\title{
Alfvén 'resonance' reconsidered: Exact equations for wave propagation across a cold inhomogeneous plasma
}

\author{
P. M. Bellan \\ California Institute of Technology, Pasadena, California 91125
}

(Received 10 March 1994; accepted 30 June 1994)

\begin{abstract}
Previous discussions of Alfvén wave propagation across an inhomogeneous plasma predicted that shear Alfvén waves become singular (resonant) at the $\omega=k_{z} v_{A}$ layer and that there is a strong wave absorption at this layer giving localized ion heating. In this paper the three standard derivations of the Alfvén 'resonance' (incompressible magnetohydrodynamics, compressible magnetohydrodynamics, and two-fluid) are re-examined and shown to have errors and be mutually inconsistent. Exact two-fluid differential equations for waves propagating across a cold inhomogeneous plasma are derived; these show that waves in an ideal cold plasma do not become 'resonant' at the Alfvén layer so that there is no wave absorption or localized heating. These equations also show that the real 'shear' Alfvén wave differs in substance from both the ideal MHD and earlier two-fluid predictions and, in the low density, high field region away from the $\omega=k_{z} v_{A}$ layer, is actually a quasielectrostatic resonance cone mode. For $\omega \ll \omega_{c i}$ and $k_{y}=0$, the $\omega=k_{z} v_{A}$ layer turns out to be a cutoff (reflecting) layer for both the 'shear' and compressional modes (and not a resonance layer). For finite $\omega / \omega_{c i}$ and $k_{y}=0$ this layer becomes a region of wave inaccessibility. For $\omega \ll \omega_{c i}$ and finite $k_{y}$ there is strong coupling between shear and compressional modes, but still no resonance.
\end{abstract}

\section{INTRODUCTION}

\section{A. The importance of the Alfuén resonance concept}

The Alfvén resonance, an ideal magnetohydrodynamic (MHD) concept, was proposed over two decades ago by Uberoi $^{1}$ and by Tataronis and Grossmann. ${ }^{2}$ In Ref. 2 the Alfvén resonance is described as a logarithmic singularity which occurs in a cold magnetized slab plasma that is inhomogeneous in the $x$ direction (the $z$ direction is parallel to the equilibrium magnetic field). The singularity occurs at the critical layer where $\omega=k_{z} v_{A}(x)$. Also in Ref. 2 it is proposed that wave phase mixing at the shear Alfvén layer will produce a strong, localized heating similar to Landau damping. Since the Alfvén resonance was originally discussed in Refs. 1 and 2 , many papers and a monograph ${ }^{3}$ have been published in which the Alfvén resonance plays a pivotal role. Examples of papers invoking the Alfvén resonance include the following:

(1) Chen and Hasegawa $a^{4,5}$ made a quantitative calculation of the heating rate due to phase mixing at the Alfvén resonance and argued that the heating rate is independent of the actual physical absorption mechanism at the resonance.

(2) Goedbloed ${ }^{6}$ discussed the spectrum of ideal MHD in axisymmetric toroidal systems.

(3) Stix $^{7.8}$ examined the Alfvén resonance using a two-fluid model with finite $\omega / \omega_{c i}$ (unlike MHD where $\omega / \omega_{c i}=0$ is assumed). The Alfvén resonance was retrieved from the two-fluid equations and mode conversions to both cold and hot plasma waves were discussed in this two fluid context.
(4) Hasegawa and $\mathrm{Chen}^{9,10}$ also predicted a mode conversion at the Alfvén resonance for a collisionless plasma, but used a kinetic modification to the MHD equations.

(5) Rosenbluth and Rutherford ${ }^{11}$ added finite ion gyroradius effects to the Alfvén resonance and found a strong interaction with hot ions.

(6) Ott, Wersinger, and Bonoli ${ }^{12}$ proposed a cavity mode heating mechanism where the magnetosonic mode interacts with the Alfvén resonance.

(7) Karney, Perkins, and Sun ${ }^{13}$ considered the effect of compressibility and finite $\omega / \omega_{c i}$ on heating at the Alfvén resonance in a large tokamak.

(8) Li, Mahajan, and Ross ${ }^{14}$ invoked the Alfvén resonance in a toroidal model of Alfvén modes and found the Alfvén resonance to be an essential part of the description of toroidal Alfvén eigenmodes.

(9) Mett and Taylor ${ }^{15}$ invoked the Alfvén 'resonance' as part of a helicity injection current drive scheme.

(10) Vaclavic and Appert ${ }^{16}$ have written an extensive review on Alfvén wave heating and discuss the central role of the Alfvén resonance.

(11) The Alfvén resonance has been investigated on: the 'Tokamak Chauffage Alfvén' 17 (TCA) in Switzerland; the Tortus ${ }^{18}$ tokamak in Australia, and the Phaedrus ${ }^{19}$ tokamak at the University of Wisconsin. Heating results on TCA have been indirectly interpreted in terms of the Alfvén resonance, but there have been no in situ measurements of fields demonstrating resonant absorption at the $\omega=k_{z} v_{A}$ layer. Both electron and ion heating were observed but there was no clear connection to the Alfvén resonance (i.e., there was no direct observation of hot ions concentrated at the $\omega=k_{z} v_{A}$ layer). In con- 
trast to the prediction by the various Alfvén resonance heating theories that there should be complete wave absorption by ions at the Alfvén layer, the TCA experiment found $^{17}$ that of $140 \mathrm{~kW}$ radio frequency (RF) power injected into the plasma only $7.6 \mathrm{~kW}$ went into ion heating.

(12) The Alfvén resonance has also been investigated in linear machines by Cekic et $a .^{20}$ and by Tsushima et al. ${ }^{21}$ The plasmas in these devices were highly collisional, i.e., $\omega \ll v_{e i}$, and so were not in the 'ideal' regime.

(13) Donnelly and Clancy ${ }^{22}$ analyzed the Alfvén resonance in a cylindrical plasma both analytically and numerically and compared their results to Ref. 21.

(14) Lee and Roberts, ${ }^{23}$ Hollweg and Yang, ${ }^{24}$ and Parker ${ }^{25}$ discuss Alfvén resonance absorption in the context of the magnetosphere and the solar corona and also predict strong plasma heating due to phase mixing and/or linear mode conversion at the Alfvén resonance layer.

(15) Thompson and Wright ${ }^{26}$ use a generalized Frobenius series to examine the details of wave hehavior at the resonance layer.

(16) Steinolfson and Davila ${ }^{27}$ have found heating at the Alfvén resonance layer using a numerical simulation of a fully compressible, low beta, resistive plasma intended to model solar coronal loops.

(17) Witherspoon, Prager, and Sprott ${ }^{28}$ measured a localized peaking of the poloidal component of the RF magnetic field in the Tokapole II tokamak and interpreted this peaking as a verification of the Alfven resonance.

From the above list we see that there have been a large number of thcoretical papers examining the conscquences of the Alfvén resonance, several experiments designed to exploit it, and a few experiments designed to measure it. A peaking of the poloidal magnetic field has been observed at the $\omega=k_{z} v_{A}$ layer in Ref. 28; this peaking was interpreted as a manifestation of the Alfvén resonance and the finite magnitude of the peak was attributed to dissipative effects. There do not exist any experimental measurements demonstrating strong wave absorption or localized ion heating at the Alfvén layer (an experiment showing strong wave absorption at the Alfvén layer would show a traveling wave propagating towards the Alfvén layer; if complete absorption indeed occurred at this layer, the wave amplitude would decrease to zero in a very short distance and the wave energy would show up in the form of locally heated ions).

\section{B. Our claim: The Alfuén resonance is an invalid concept}

We claim that the prediction that a cold plasma has an 'Alfvén resonance' is spurious and show that cold plasma Alfvén waves are not singular at the $\omega=k_{z} v_{A}(x)$ layer. Thus, phase mixing and wave absorption (dissipation) do not occur in a cold collisionless plasma at the Alfvén layer. Consequently, the elaborate mathematical technique ${ }^{2,3}$ devised to deal with the singularity is unnecessary and irrelevant. The incorrect prediction that cold plasma waves phase mix and are absorbed at the Alfvén resonance constitutes a significant and important failure of ideal MHD, traditionally considered an accurate model of plasma behavior at low frequencies (i.e., $\omega \ll \omega_{c i}$, where $\omega_{c i}$ is the ion cyclotron frequency).

Instead of being a critical resonant layer where singularities and complicated physical effects occur, we show instead that the $\omega=k_{z} v_{A}(x)$ layer (in the simplest situation) is just a cutoff for both of the two normal modes that exist in a cold plasma. Both modes are perfectly well behaved at this layer. In more complicated situations (which we shall also discuss) the two modes are coupled, but there is still no singularity or absorption.

This paper is organized as follows: Sec. II gives a detailed review of the standard Alfven modes and of the three different ways the Alfvén resonance has been derived (incompressible MHD, compressible MHD, two fluid). In Sec. III we re-examine these three derivations of the Alfvén resonance, point out errors, and show that the three models are mutually inconsistent. In Sec. IV we use two fluid theory and the complete Maxwell equations (not the MHD approximation) to derive exact partial differential equations valid for arbitrary frequencies for a wave propagating in an inhomogeneous cold magnetized plasma; these equations transcend ideal MHD. Section V discusses these equations when $k_{y}=0$ and Sec. VI discusses what happens when $k_{y}$ is finite; although the $k_{y}=0$ and $k_{y} \neq 0$ situations differ significantly, in both cases it is shown that there is no singularity at the $\omega=k_{z} v_{A}(x)$ layer. In Sec. $V$ it is also shown that the shear Alfvén mode turns out to be a quasielectrostatic wave except in the immediate vicinity of the Alfvén layer, while Sec. VI also shows that if $k_{y}$ is finite there is a strong coupling between shear and compressional modes at the $\omega=k_{z} v_{A}(x)$ layer. Section VII shows that when the broad $k_{z}$ spectrum excited by a finite extent source is taken into account, the quasielectrostatic shear mode has a resonance cone behavior in regions where $k_{z} v_{A}(x) \gg \omega$. Section VIII discusses a possible toroidal current drive scheme based on Landau damping of the quasielectrostatic shear mode. Section IX contains a summary and conclusions.

\section{REVIEW OF ALFVÉN MODES AND DERIVATIONS OF THE ALFVÉN 'RESONANCE'}

We now present a brief, but detailed review of $\mathrm{MHD}$ Alfvén waves and the various models for the Alfvén 'resonance'; the point of this review is to clarify all assumptions and approximations so that errors and inconsistencies in these assumptions/approximations can be identified in Secs. III and VI. We will use direction conventions and nomenclature consistent with the standard notation introduced by Stix; ${ }^{29}$ parallel and perpendicular are with respect to the equilibrium magnetic field $\mathbf{B}=B \hat{z}$.

\section{A. Shear mode propagating exactly in the $z$ direction (original Alfvén analyses)}

In 1943 Alfvén $^{30}$ described what he called electromagnetic-hydrodynamic waves and what are now called shear (or torsional) Alfvén waves. Alfvén considered plane waves propagating exactly in the $z$ direction in a magnetized perfectly conducting fluid; i.e., he assumed that the wave had 
a space-time dependence $\sim \exp \left(i k_{z} z-i \omega t\right)$. Thus, this derivation implicitly assumes that $k_{x}=k_{y}=0$. Alfvén's derivation combined the linearized equation of motion

$$
-i \omega \rho \tilde{\mathbf{U}}=\tilde{\mathbf{J}} \times B \hat{z}-\nabla \tilde{P},
$$

Ohm's law for a perfectly conducting fluid,

$$
\overline{\mathbf{E}}+\overline{\mathbf{U}} \times B \hat{z}=0,
$$

Faraday's law

$$
\nabla \times \tilde{\mathbf{E}}=i \omega \tilde{\mathbf{B}},
$$

and Ampère's law

$$
\nabla \times \overline{\mathbf{B}}=\mu_{0} \tilde{\mathbf{J}} .
$$

He assumed that the conducting fluid was incompressible

$$
\boldsymbol{\nabla} \cdot \tilde{\mathbf{U}}=0
$$

so that $\tilde{U}_{z}=0$ and also assumed that $\tilde{J}_{y}=\tilde{J}_{z}=0$ so that $\bar{U}_{x}=0$. Thus, the only finite velocity component is $\tilde{U}_{y}$ in which case Eq. (1) gives

$$
\tilde{U}_{y}=\frac{\tilde{J}_{x} B}{i \omega \rho} .
$$

Ohm's, Faraday's and Ampère's laws respectively yield

$$
\tilde{E}_{x}=-\tilde{U}_{y} B, \quad k_{z} \tilde{E}_{x}=\omega \tilde{B}_{y}, \quad i k_{z} \tilde{B}_{y}=-\mu_{0} \tilde{J}_{x} .
$$

These constitute four equations in the four unknowns, $\tilde{E}_{x}$, $\tilde{U}_{y}, \tilde{J}_{x}$, and $\tilde{B}_{y}$. The determinant of this system of equations gives the dispersion relation

$$
\omega^{2}=k_{z}^{2} v_{A}^{2}
$$

where

$$
v_{A}=\frac{B}{\sqrt{\mu_{0} \rho}}
$$

is the Alfven velocity. Although this derivation is elementary, we have repeated it here to stress three points which will be important later, namely:

(1) the plane wave was assumed to be exactly in the $z$ direction (i.e., $k_{x}=k_{y}=0$ ),

(2) this mode involves a shearing, incompressible motion, since the velocity is in the $y$ direction while the propagation is in the $z$ direction,

(3) if we assume that the only finite perturbed quantities are $\tilde{E}_{x}, \bar{U}_{y}, \bar{J}_{x}$, and $\tilde{B}_{y}$, it is then unnecessary to assume the fluid is incompressible, since the dynamics automatically give incompressibility. Thus, Eq. (5) is redundant once we have chosen the 'eigenvector' $\left\{\tilde{E}_{x}, \tilde{U}_{y}, \tilde{J}_{x}\right.$, $\left.\tilde{B}_{y}\right\} \neq 0$. It is the wave that is incompressible and not the plasma.

\section{B. Extension of MHD to arbitrary propagation direction}

Shortly after Alfvén's paper appeared, Astrom $^{31}$ and Dungy ${ }^{32}$ considered wave propagation in an arbitrary direction; i.e., a perturbation having the dependence $\exp (i \mathbf{k} \cdot \mathbf{x}-i \omega t)$ was assumed where the wavevector $\mathbf{k}=k_{x} \hat{x}+k_{y} \hat{y}+k_{z} \hat{z}$ was arbitrary. References 31 and 32 found two linearly independent orthogonal modes in the low frequency MHD regime (i.e., $\omega \ll \omega_{c i}$ ); these modes are now commonly called the shear and compressional Alfvén modes. Without loss of generality the coordinate system is oriented so that the $x$ direction is parallel to $\mathbf{k}_{1}$; thus by choice of coordinate system, $k_{y}=0$. The eigenvectors for the two MHD modes are categorized in Eq. (10). The set of field components associated with the compressional mode are boxed; the set of unboxed components constitute the eigenvector for the shear mode (with the exception of the special case of $\tilde{E}_{z}$ which is zero for both modes)

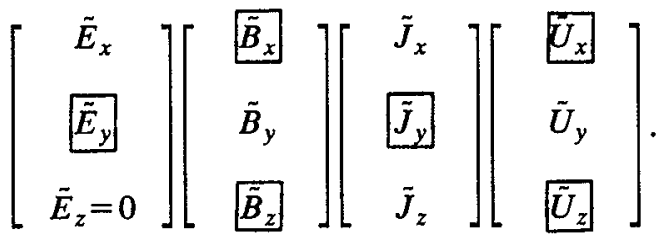

The condition $\tilde{E}_{z}=0$ is a fundamental property of ideal MHD and is intrinsic to all ideal MHD theories.

\section{Shear Alfvén wave with arbitrary propagation direction}

Using the unboxed components, i.e., $\tilde{E}_{x}, \tilde{B}_{y}, \tilde{J}_{x}, \tilde{J}_{z}$, and $\tilde{U}_{y}$, Eqs. (1)-(4) become

$$
\begin{aligned}
& -i \omega \rho \tilde{U}_{y}=-\tilde{J}_{x} B, \quad \tilde{E}_{x}+\tilde{U}_{y} B=0, \quad i k_{z} \tilde{E}_{x}=i \omega \tilde{B}_{y}, \\
& -i k_{z} \tilde{B}_{y}=\mu_{0} \tilde{J}_{x}, \quad i k_{x} \tilde{B}_{y}=\mu_{0} \tilde{J}_{z} .
\end{aligned}
$$

The first four of these five equations constitute four coupled homogeneous equations in the four unknowns $\tilde{E}_{x}, \tilde{B}_{y}, \tilde{J}_{x}$, and $\tilde{U}_{y}$ and the determinant of this system of four equations gives the shear Alfvén dispersion relation

$$
\omega^{2}=k_{z}^{2} v_{A}^{2}
$$

which is identical to Alfvén's original result, except that here $k_{x}$ is arbitrary rather than zero. The fifth equation simply specifies $\tilde{J}_{z}$ once the other four quantities have been determined.

Astrom first pointed out a peculiar feature of Eq. (12): even though $k_{x}$ is finite it does not appear in Eq. (12). This unusual property has been interpreted to mean that shear wave disturbances started on a given magnetic field line propagate along that field line, and do not spread out from that line. Hence, disturbances on adjacent field lines are 'insulated' from each other. To quote Astrom "31 "the energy is propagated only along the (equilibrium) lines of force, but the wave normal may have an arbitrary direction"; to quote Stix $^{33}$ "a wave packet comprised of a spectrum of $k_{x}$ values will then propagate through a uniform plasma without defocusing or dispersion, and the propagation along each line of 
force will be independent of the propagation of waves along adjacent lines of force." Similar statements have been made by other authors.

\section{Compressional Alfvén wave}

By using the eigenvector corresponding to the boxed components in Eq. (10) and assuming a cold plasma, the equation of motion, Ohm's law, Faraday's law, and Ampère's law become

$-i \omega \rho \tilde{U}_{x}=\tilde{J}_{y} B, \quad \tilde{U}_{z}=0, \quad \tilde{E}_{y}-\tilde{U}_{x} B=0$,

$-i k_{z} \tilde{E}_{y}=i \omega \tilde{B}_{x}, \quad i k_{x} \tilde{E}_{y}=i \omega \tilde{B}_{z}, \quad i k_{z} \tilde{B}_{x}-i k_{x} \tilde{B}_{z}=\mu_{0} \tilde{J}_{y}$.

The determinant of this system of equations gives the cold plasma dispersion

$$
\omega^{2}=\left(k_{x}^{2}+k_{z}^{2}\right) v_{A}^{2} .
$$

This mode is called the compressional mode since $\boldsymbol{\nabla} \cdot \tilde{\mathbf{U}}=i k_{x} \tilde{U}_{x}$ is nonzero. If finite pressure is included, as for a warm plasma, then a sound wave appears associated with finite $\tilde{U}_{z}$; we will not consider this sound wave since we are restricting our discussion to cold plasma waves.

\section{Incompressible MHD derivation of the Alfuén resonance}

The Alfvén resonance concept was independently proposed in Refs. 1 and 2; the analyses in these papers assume an incompressible plasma, i.e., $\boldsymbol{\nabla} \cdot \tilde{\mathbf{U}}=0$ (we note that the same basic equations were discussed more recently in Ref. 23). The essential result of Refs. 1 and 2 is that the peculiar lack of dependence of $\mathrm{Eq}$. (12) on $k_{x}$ (i.e., lack of dispersion in the $x$ direction) is removed by considering a plasma that is inhomogeneous in the $x$ direction. By taking this inhomogeneity into account, Refs. 1 and 2 show that Eq. (12) becomes a resonance condition (singularity in a wave equation), rather than a dispersion relation; the location where $\omega=k_{z} v_{A}(x)$ is called the Alfvén resonance layer. This resonance is specifically identified with the shear mode, since the resonance condition is identical to Eq. (12) and also since the mode is incompressible (as is the shear mode).

We now give a brief outline of the derivation presented in Refs. 1 and 2 for the Alfvén resonance. All perturbations are assumed to be of the form $f(x) \exp \left(i k_{z} z-i \omega t\right)$. An equilibrium pressure gradient exists in the $x$ direction so that the equilibrium magnetic field depends on $x$ such that

$$
P(x)+\frac{B^{2}(x)}{2 \mu_{0}}=\text { const. }
$$

We define the perturbed fluid position $\tilde{\xi}=i \tilde{\mathrm{U}} / \omega$ so that the combination of Ohm's and Faraday's laws give

$$
\tilde{B}=\nabla \times(\tilde{\xi} \times \mathbf{B})
$$

which has components

$$
\tilde{B}_{x}=i k_{z} B \tilde{\xi}_{x}, \quad \tilde{B}_{y}=i k_{z} B \tilde{\xi}_{y}, \quad \tilde{B}_{z}=-\frac{d}{d x}\left(B \tilde{\xi}_{x}\right) .
$$

The linearized equation of motion is

$$
-\omega^{2} \mu_{0} \rho \tilde{\xi}=-\nabla \tilde{p}+\mathbf{B} \cdot \nabla \tilde{\mathbf{B}}+\tilde{\mathbf{B}} \cdot \nabla \mathbf{B}
$$

where

$$
\tilde{p}-\mu_{0} \tilde{P}+\mathbf{B} \cdot \tilde{\mathbf{B}} .
$$

Using Eq. (17), the components of Eq. (18) are found to be

$$
\begin{aligned}
& -\omega^{2} \mu_{0} \rho \tilde{\xi}_{x}=-\frac{d \tilde{p}}{d x}-k_{z}^{2} B^{2} \tilde{\xi}_{x}, \\
& -\omega^{2} \mu_{0} \rho \tilde{\xi}_{y}=-k_{z}^{2} B^{2} \tilde{\xi}_{y}, \\
& -\omega^{2} \mu_{0} \rho \tilde{\xi}_{z}=-i k_{z} \tilde{p}-i k_{z} B \frac{d}{d x}\left(B \tilde{\xi}_{x}\right)+i k_{z} B \tilde{\xi}_{x} \frac{d B}{d x} .
\end{aligned}
$$

The $y$ component is decoupled from the $x$ and $z$ components and is not used, while the $x$ component is re-written as

$$
\frac{d \tilde{p}}{d x}=\left(\omega^{2} \mu_{0} \rho-k_{z}^{2} B^{2}\right) \tilde{\xi}_{x}
$$

and the $z$ component becomes

$$
i k_{z} \tilde{p}=\omega^{2} \mu_{0} \rho \tilde{\xi}_{z}-i k_{z} B^{2} \frac{d \tilde{\xi}_{x}}{d x} .
$$

At this point, the incompressibility assumption is invoked to give

$$
\frac{d \tilde{\xi}_{x}}{d x}+i k_{z} \tilde{\xi}_{z}=0
$$

this is used to eliminate $\xi_{z}$ from Eq. (24) which becomes

$$
k_{z}^{2} \tilde{p}=\left(\omega^{2} \mu_{0} \rho-k_{z}^{2} B^{2}\right) \frac{d \tilde{\xi}_{x}}{d x} .
$$

This is used to eliminate $\tilde{p}$ in Eq. (23) to give

$$
\frac{d}{d x}\left[\left(\omega^{2} \mu_{0} \rho-k_{z}^{2} B^{2}\right) \frac{d \tilde{\xi}_{x}}{d x}\right]-k_{z}^{2}\left(\omega^{2} \mu_{0} \rho-k_{z}^{2} B^{2}\right) \tilde{\xi}_{x}=0 .
$$

The quantity $\epsilon(x)$ is defined as

$$
\epsilon(x)=\omega^{2} \mu_{0} \rho-k_{z}^{2} B^{2}(x)=\left(\omega^{2}-k_{z}^{2} v_{A}^{2}\right) \mu_{0} \rho
$$

and the Alfvén resonance is located where $\epsilon(x)=0$, i.e., where $\omega^{2}=k_{z}^{2} v_{A}^{2}(x)$. If we re-define the $x$ origin to be at this point, then in the neighborhood of $x=0$, we can Taylor expand

$$
\epsilon(x)=x \epsilon^{\prime}
$$

so that Eq. (27) becomes

$$
\frac{d}{d x}\left[x \frac{d \tilde{\xi}_{x}}{d x}\right]-k_{z}^{2} x \tilde{\xi}_{x}=0
$$

which has solutions $I_{0}\left(k_{z} x\right)$ and $K_{0}\left(k_{z} x\right)$, the modified Bessel functions of order zero. For small argument $K_{0}\left(k_{z} x\right)$ contains a term $\sim \ln x$; this Iogarithmic singularity is the essence of the "Alfvén resonance" described in Refs. 1 and 2. It 
is interesting to note that the actual value of $\tilde{P}$ did not matter, and so this model is valid for cold as well as warm plasmas. This basic model has been extended by the original authors and by others to include finite $k_{y}$, sheared magnetic fields, toroidal geometry, flux coordinates, etc. (see list of examples in Sec. I).

\section{Alfuén resonance with compressible MHD}

References 3 and 4 discussed a similar model for the Alfvén resonance, but with the important difference that the plasma was allowed to be compressible. The adiabatic relation between the perturbed pressure and velocity,

$$
\tilde{P}=-\gamma P \nabla \cdot \tilde{\xi}-\tilde{\xi} \cdot \nabla P
$$

was used to eliminate $\tilde{P}$ in Eq. (19). Also finite $k_{y}$ was assumed. With these assumptions, and using manipulations similar to those for the incompressible case, Refs. 3 and 4 give in the cold plasma limit a compressible wave equation of the form

$\frac{d}{d x}\left[\frac{\left(\omega^{2} \rho \mu_{0}-k_{z}^{2} B^{2}\right) B^{2}}{\left(\omega^{2} \rho \mu_{0}-\left(k_{y}^{2}+k_{z}^{2}\right) B^{2}\right)} \frac{d \tilde{U}_{x}}{d x}\right]+\left(\omega^{2} \rho \mu_{0}-k_{z}^{2} B^{2}\right) \tilde{U}_{x}=0$.

Hasegawa and Uberoi ${ }^{34}$ state that Eq. (32) "is a generalized form of Eq. (27) in that the effect of compressibility is included" (equations have been renumbered to refer to numbering system here).

\section{E. Two-fluid derivation of Alfvén resonance}

The MHD equations are a less precise description of the plasma than are the two-fluid equations. In Ref. 29 the twofluid cold plasma wave equations were discussed in detail and, in particular, a useful notation for the cold plasma dielectric tensor $\mathrm{K}$ was introduced, namely

$$
\mathrm{K}=\left[\begin{array}{ccc}
S & -i D & 0 \\
i D & S & 0 \\
0 & 0 & P
\end{array}\right]
$$

with elements

$$
\begin{aligned}
& S=1-\sum_{\sigma=i, e} \frac{\omega_{p \sigma}^{2}}{\omega^{2}-\omega_{c \sigma}^{2}}, \quad D=\sum_{\sigma=i, e} \frac{\omega_{c \sigma} \omega_{p \sigma}^{2}}{\omega\left(\omega^{2}-\omega_{c \sigma}^{2}\right)}, \\
& P=1-\sum_{\sigma=i, e} \frac{\omega_{p \sigma}^{2}}{\omega^{2}} .
\end{aligned}
$$

Using this notation, the cold plasma wave equation has the form

$$
\boldsymbol{\nabla} \times \nabla \times \tilde{\mathbf{E}}+\frac{\omega^{2}}{c^{2}} \mathrm{~K} \cdot \tilde{\mathbf{E}}=\mathbf{0} .
$$

By assuming an $\exp (i \mathbf{k} \cdot \mathbf{x})$ spatial dependence, defining the refractive index vector $\mathbf{n}=c \mathbf{k} / \omega$, and choosing the $x$ axis to lie along $\mathbf{n}_{\perp}$, Eq. (35) can be expressed as

$$
\left[\begin{array}{ccc}
S-n_{z}^{2} & -i D & n_{x} n_{z} \\
i D & S-n^{2} & 0 \\
n_{x} n_{z} & 0 & P-n_{x}^{2}
\end{array}\right] \cdot\left[\begin{array}{c}
\tilde{E}_{x} \\
\tilde{E}_{y} \\
\tilde{E}_{z}
\end{array}\right]=0
$$

This system of equations has nontrivial solutions (normal modes) when the determinant of the matrix vanishes. By defining $\theta$ as the angle between the refractive index and the equilibrium magnetic field so that

$$
n_{x}=n \sin \theta, \quad n_{z}=n \cos \theta
$$

and defining the auxiliary quantities

$$
R=S+D, \quad L=S-D
$$

the vanishing of the determinant can be expressed simply as

$$
\mathscr{C} n^{4}-\mathscr{B} n^{2}+\mathscr{C}=0
$$

where

$$
\begin{aligned}
& \mathscr{A}=S \sin ^{2} \theta+P \cos ^{2} \theta, \\
& \mathscr{B}=R L \sin ^{2} \theta+P S\left(1+\cos ^{2} \theta\right), \\
& \mathscr{C}=P R L .
\end{aligned}
$$

In Ref. 8 it was argued that since $|P| \rightarrow \infty$ as $\omega \rightarrow 0$, then in both the ion cyclotron frequency regime where $\omega \approx \omega_{c i}$ and also in the MHD-like regime where $\omega \ll \omega_{c i}$, the quantities $\mathscr{A}, \mathscr{S}, \mathscr{C}$ in Eq. (40) can be approximated as

$$
\mathscr{A} \simeq P \cos ^{2} \theta, \quad \mathscr{B} \simeq P S\left(1+\cos ^{2} \theta\right), \quad \mathscr{C} \simeq P R L
$$

provided $\theta$ is not too close to $\pi / 2$ so that $\left|S \sin ^{2} \theta\right|$ $\ll \mid P \cos ^{2} \theta$. In this case, the quantity $P$ factors from Eq. (39), which becomes simply

$$
n^{4} \cos ^{2} \theta-n^{2} S\left(1+\cos ^{2} \theta\right)+R L=0
$$

or in terms of $n_{x}$ and $n_{z}$

$$
n_{x}^{2}\left(n_{z}^{2}-S\right)-2 S n_{z}^{2}+n_{z}^{4}+R L=0
$$

which may be solved exactly for $n_{x}^{2}$ to give

$$
n_{x}^{2}=\frac{\left(n_{z}^{2}-R\right)\left(n_{z}^{2}-L\right)}{S-n_{z}^{2}} .
$$

Reference 8 states "the form of the dispersion Eq. (44) reveals the resonance, called the Alfvén resonance, that occurs under the condition $S=n_{z}^{2}$; this resonance results in $n_{x}^{2} \rightarrow \infty$. That this corresponds to the Alfvén resonance discussed in Refs. 1 and 2 can be seen by noting that at low frequencies $S$ has the limiting form $S=1+c^{2} / v_{A}^{2}$. Thus, if $v_{A} \ll c$, this resonance for $n_{x}^{2}$ occurs when $\omega=k_{z} v_{A}$ and so the Alfvén 'resonance' in Ref. 8 is interpreted as being the same as that of Refs. 1 and 2. Equation (44) has also been analyzed in Ref. 13. Both Refs. 8 and 13 discuss the peculiar cutoff-resonance-cutoff 'triplet' form of Eq. (44) where the $n_{z}^{2}=S$ Alfvén resonance is tightly sandwiched in between the $n_{z}^{2}=R$ and $n_{z}^{2}=L$ cutoffs. 


\section{F. Summary of claimed properties of the Alfvén resonance}

For reference, we summarize the previously claimed properties of the Alfvén resonance and cite where these properties were discussed:

Previous claim \# 1 . In the cold plasma approximation, the MHD velocity $\tilde{U}_{x}$ is logarithmically singular ${ }^{2,4,9,10,12,23}$ at the Alfvén layer $\omega=k_{z} v_{A}(x)$.

Previous claim \#2. There is a resonant absorption ${ }^{2,3,5,12}$ of the logarithmically singular wave at the Alfvén layer; this resonant absorption results from wave phase mixing (analogous to Landau damping). As discussed in detail on pages 49-54 of Ref. 3 this phase mixing is calculated for Eq. (27) using an elaborate mathematical procedure, which we summarize briefly: the $x$ origin is chosen so that $x=0$ is the location of the singular layer. The plasma is assumed to have a linearly increasing density for $|x|<a$ and a constant density for $|x|>a$. The modified Bessel function solutions found in the region $|x|<a$ are matched at $|x|=a$ to exponential external solutions existing for $|x|>a$. This matching results in an expression $\left[D\left(k_{z}, \omega\right)\right.$ in the notation of Ref. 3], involving the 'conjunct' (related to the Wronksian) of Eq. (27); $D\left(k_{z}, \omega\right)$ is interpreted as a 'dispersion relation'. Because of the logarithmic terms this 'dispersion' is multivalued in the complex plane so that a Reimann surface and branch cuts must be introduced to resolve the multivalued ambiguity of the logarithm. Using the Nyquist criterion, it is argued that $D\left(k_{z}, \omega\right)$ has no zeros on the principle sheet, but does have zeros on the other sheets of the Reimann surfacc. The zero of $D\left(k_{z}, \omega\right)$ is calculated for the sheet displaced one Reimann level from the principal sheet and it is found that this root gives a complex $\omega$ which is interpreted as a spatially localized damping which gives resonant absorption. It is argued that this damping is analogous to the phase mixing involved in Landau damping.

\section{WHY THE ALFVÉN RESONANCE THEORIES ARE INCORRECT}

We now show that both the incompressible MHD derivation and the two-fluid derivation for the Alfvén resonance discussed above are incorrect. The interpretation of the compressible MHD derivation is more involved and will be deferred to Secs. V and VI.

\section{A. The error in the derivation of the incompressible MHD model of the Alfvén resonance}

We showed in Sec. II B 1 that the uniform plasma shear mode is incompressible and in Sec. II B 2 that the compressional mode is compressible. Further, we listed the eigenvectors for the shear and incompressible modes in Eq. (10). We show here that the essential error in Refs. 1 and 2 is that incompressibility is prescribed for the eigenvectors belonging to the compressional mode. In other words, the compressional mode is artificially forced to be incompressible.

This is most casily seen for the case of a cold plasma where $\tilde{P}=0$ so that

$$
\tilde{p}=\mathbf{B} \cdot \tilde{\mathbf{B}} \text {. }
$$

Mathematically, the error involves overdetermining the system of equations by invoking the incompressibility condition Eq. (25) to solve for $\tilde{p}$ in Eq. (26). This step is wrong because it is incorrect to invoke incompressibility to find $\tilde{p}$ since $\tilde{p}$ is already completely determined as can be seen by substituting Eq. (17) into Eq. (45) (if the plasma has finite pressure, then $\tilde{P}$ and hence $\tilde{p}$ can be found in terms of $\xi$, so the problem is still overdetermined).

\section{B. Inconsistency between the incompressible and compressible MHD models of the Alfvén resonance}

In Ref. 34 it was stated that Eq. (32) is a gencralization of Eq. (27) such that compressibility is included. In fact, Eq. (32) is not at all a generalization of Eq. (27) since if we set $k_{y}=0$ in order to correspond to Eq. (27), then Eq. (32) simplifies to

$$
\frac{d}{d x}\left[B^{2} \frac{d \tilde{U}_{x}}{d x}\right]+\left(\omega^{2} \rho \mu_{0}-k_{z}^{2} B^{2}\right) \tilde{U}_{x}=0
$$

which is clearly different from Eq. (27). If we assume that the plasma is uniform in the $x$ direction, then we may Fourier analyze in the $x$ direction, i.e., replace $d / d x \rightarrow i k_{x}$ so that Eq. (46) reverts to the compressional Alfven dispersion, Eq. (14). Thus, if $k_{y}=0$, Eq. (46) describes compressional modes propagating in an inhomogeneous plasma.

When $k_{y}$ is finite, Eq. (32) still predicts a resonance behavior; we shall show in Sec. VI B that this predicted resonance is also spurious.

\section{Errors in the phase-mixing argument}

We shall show later that there is no logarithmic singularity and so the entire phase mixing argument is unnecessary. Nevertheless, it is worthwhile pointing out that the phase mixing argument is not appropriate to a real experimental situation where an antenna having a fixed $k_{z}$ is driven by a steady-state oscillator having a fixed frequency $\omega$. Thus, both $k_{z}$ and $\omega$ are prescribed and real (as distinct from complex) quantities. In this steady-state situation, all linearly perturbed quantities will also oscillate at the transmitter frequency $\omega$ and will also have the antenna parallel wave number $k_{z}$. It is mathematically incorrect to assign an imaginary part to $\omega$ for this steady-state situation; yet the essence of the phasemixing argument is that $\omega$ becomes complex. In a steadystate situation, spatially localized absorption would be manifested by $k_{x}$ developing a large imaginary part in the absorption region.

\section{Inconsistency between the two-fluid Alfvén 'resonance' and the MHD Alfvén resonances}

Both incompressible and compressible MHD models predict an Alfvén resonance in the MHD limit, i.e., when $\omega / \omega_{c i} \rightarrow 0$. The incompressible model (which we showed involved overdetermining the system of equations) gave a resonance when $k_{y}$ was zero, but the compressible model, Eq. (32), requires finite $k_{y}$ to have a resonance. The two-fluid model, Eq. (44), predicts a resonance for $k_{y}=n_{y} \omega / c=0$ (so 


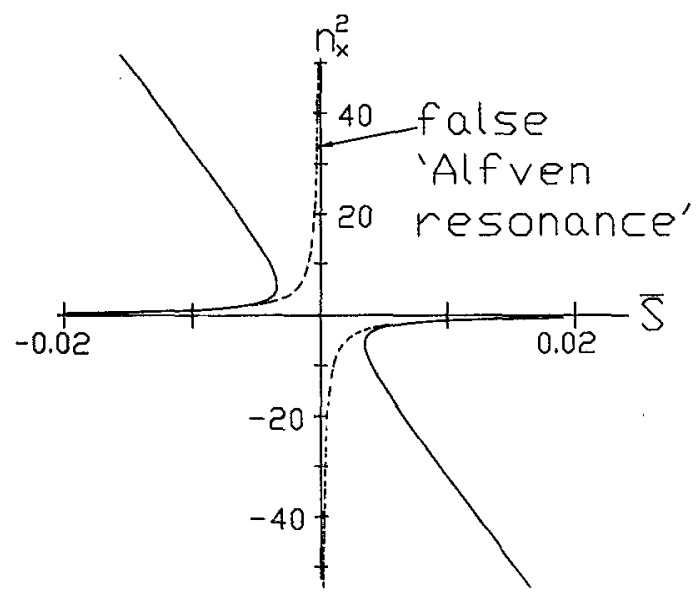

FIG. 1. $n_{x}^{2}$ vs $\bar{S}=S-n_{z}^{2}$ for $P=-10^{4}, R=3.1, L=2.9$ (ion/electron mass ratio $=12.5$ used). Solid curve is exact cold plasma solution given by Eq. (53), dashed curve is approximate solution given by Eq. (44) which correctly describes fast mode (small root) away from $\bar{S}=0$, but as $\bar{S} \rightarrow 0$ predicts the false 'Alfvén resonance'.

it is inconsistent with the compressible MHD model), and furthermore, the two-fluid 'resonance' disappears in the limit $\omega / \omega_{c i} \rightarrow 0$, since in this limit $S=R=L$ and Eq. (44) becomes simply $n_{x}^{2}=S-n_{z}^{2}$. Thus, the two-fluid 'resonance' is inconsistent with the MHD 'resonances'.

\section{E. Errors in the two-fluid prediction of the Alfven resonance}

Let us now re-examine the two-fluid prediction ${ }^{8}$ of the Alfvén resonance, i.e., Eq. (44). The exact dispersion relation, Eq. (39), can be rearranged to give a quadratic equation for $n_{x}^{2}$,

$$
S n_{x}^{4}-\left[\bar{S}(S+P)-D^{2}\right] n_{x}^{2}+P\left[\bar{S}^{2}-D^{2}\right]=0
$$

where we have defined

$$
\bar{S}=S-n_{z}^{2} .
$$

If the two roots of Eq. (47) are well separated, they consist of a big root where the first two terms balance, i.e.,

$$
n_{x}^{2}=\frac{\bar{S}(S+P)-D^{2}}{S} \quad \text { (big root) }
$$

or in the limit of large $P$,

$$
n_{x}^{2} \simeq \frac{\bar{S} P}{S} \quad \text { (big root) }
$$

The small root is found by balancing the last two terms of Eq. (47), giving

$$
n_{x}^{2} \simeq \frac{P\left[\bar{S}^{2}-D^{2}\right]}{\left[\bar{S}(S+P)-D^{2}\right]} \quad \text { (small root) }
$$

or in the limit of large $P$,

$$
n_{x}^{2} \simeq \frac{\left(n_{z}^{2}-R\right)\left(n_{z}^{2}-L\right)}{S-n_{z}^{2}} \quad \text { (small root) }
$$

which is just Eq. (44). Since, by assumption Eq. (52) is the small root, Eq. (52) becomes invalid when $S \rightarrow n_{z}^{2}$, because then the predicted $n_{x}^{2}$ diverges and so is clearly not small. Thus, the apparent Alfvén 'resonance' at $S=n_{z}^{2}$ in Eq. (44) is spurious.

The exact dispersion relation, Eq. (47), has the two roots

$$
n_{x}^{2}=\frac{\bar{S}(S+P)-D^{2} \pm \sqrt{\left[\bar{S}(S-P)-D^{2}\right]^{2}+4 P D^{2} n_{z}^{2}}}{2 S}
$$

this explicitly shows that $n_{x}^{2}$ does not have a resonance when $S=n_{z}^{2}$ and, in fact, only has a resonance when $S=0$, corresponding to the standard lower and upper hybrid resonances. Figure 1 plots the dependence on $S-n_{z}^{2}$ of the two roots of Eq. (53) for $R=3.1, L=2.9, P=-10^{4}$ (these have been chosen to give $S=3$ and $D=0.1$ corresponding to the MHD, i.e., $D \rightarrow 0$ regime). For comparison, Fig. 1 also shows as a dashed line the incorrect behavior (false resonance) predicted by Eq. (44) for the same values of $R, L$, and $P$.

\section{F. Accessibility}

It is clear from Fig. 1 that there is no resonance at $S=n_{z}^{2}$ (thus, there is no cutoff-resonance-cutoff 'triplet') and that furthermore, the region around $S=n_{z}^{2}$ is actually a region of inaccessibility analogous to the inaccessible region discussed in Refs. 35 and 36 for the context of the lower hybrid wave. In the limit $D \rightarrow 0$, this inaccessible region becomes infinitesimal in width and becomes a cutoff layer.

While more aesthetic than Eq. (53), the form of Eq. (39) tends to give the misleading impression that $\mathscr{A}$ and $\mathscr{B}$ (like $8)$ are always pure real coefficients. Adding to this is the fact that if the angle $\theta$ is real, then as shown in Refs. 29 and 31 , the quantity $\mathscr{B}^{2}-4 A B$ is never negative, so that $n^{2}$ is a pure real quantity. However, in actual fact, both $\theta$ and $n^{2}$ can indeed be complex; this occurs when the quantity

$$
\Gamma=\left[\bar{S}(S-P)-D^{2}\right]^{2}+4 P D^{2} n_{z}^{2}
$$

is negative, and corresponds to regions of inaccessibility. The regions of inaccessibility for a given $n_{z}$ can be visualized by plotting the regions where $\Gamma$ is negative on a ClemmowMullaly-Allis (CMA) diagram. ${ }^{37}$ The only possible negative term in $\Gamma$ is the term containing $P$; thus negative $\Gamma$ is only possible when $P<0$. This occurs for frequencies below the plasma frequency (i.e., to the right of the $P=0$ line in the CMA diagram). When $D$ is finite, mode conversion will occur in the inaccessible region.

\section{G. Demonstration that the MHD shear Alfvén wave violates quasineutrality if $k_{x} \neq 0$}

We have shown the failure of the attempts to extend Alfvén's original result to situations where the wave has an $x$ dependence. What about Eq. (12) which looks like Alfvén's 
original result, but allows $k_{x}$ to be arbitrary? We will show here that Eq. (12) is inconsistent with the assumptions of ideal MHD.

One of the fundamental assumptions of ideal MHD is that the plasma can be described by the pre-Maxwellian form of Ampère's law, i.e., by Eq. (4). An immediate consequence of this assumption is obtained by taking the divergence of Eq. (4) to obtain

$$
\boldsymbol{\nabla} \cdot \tilde{\mathbf{J}}=\mathbf{0} \text {. }
$$

The equation of charge continuity is

$$
-i \omega\left(\sum_{\sigma=i, e} \tilde{n}_{\sigma} q_{\sigma}\right)+\nabla \cdot \tilde{\mathbf{J}}=\mathbf{0}
$$

so that an essential condition for ideal MHD to be a selfconsistent approximation is that the plasma dynamics under consideration must have no net charge density, i.e.

$$
\sum_{\sigma=i, e} \tilde{n}_{\sigma} q_{\sigma}=0
$$

Now let us look at the eigenvector associated with Eq. (12) again [cf. unboxed terms in Eq. (10)]. Poisson's equation (which is omitted in MHD, but which still has to be satisfied by the real plasma) is

$$
\boldsymbol{\nabla} \cdot \tilde{\mathbf{E}}=\frac{1}{\epsilon_{0}} \sum_{\sigma=i, e} \tilde{n}_{\sigma} q_{\sigma} .
$$

Using the shear wave eigenvector, the left hand side of this equation is $i k_{x} \tilde{E}_{x}$ which is nonzero if $\tilde{E}_{x}$ is finite (which it must be since $\tilde{E}_{x}$ is part of the shear wave eigenvector) and if $k_{x}$ is finite.

Thus, when $k_{x}$ is nonzero we have a contradiction for the shear mode described by Eq. (12): the left hand side of Eq. (58) is nonzero (from direct calculation), whereas the right hand side is supposed to be zero (from the pre-Maxwellian form of Ampere's law). The only way out of this paradox is to insist that $k_{x}=0$ in which case Eq. (12) reverts to Alfvén's original form. In general, $k_{x}$ is finite and so MHD cannot provide an adequate description of the cold plasma shear mode.

\section{EXACT ARBITRARY FREQUENCY COLD MAGNETIZED PLASMA WAVE EQUATIONS}

We shall now develop an exact description of cold plasma wave propagation across an inhomogeneous plasma. Aside from incorporating the cold plasma assumption these equations involve no other assumptions, i.e., there are no restrictions on $\omega, k_{y}, k_{z}$, or density profile. Similar, but less general, equations have been previously discussed by Bers ${ }^{38}$ and by Swanson ${ }^{39}$ in the context of waveguide modes, i.e., modes propagating in the $z$ direction in a uniform plasma enclosed by a perfectly conducting closed wall. Bers' discussion was for arbitrary frequency waves, while Swanson's was restricted to low frequency waves $\left(\omega \ll \omega_{c i}\right)$ in a uniform plasma. Bers' and Swanson's waveguide results were considered as being peculiar to the waveguide geometry, and no attempt was made to apply the waveguide results to the general case of a plasma not surrounded by a perfectly conducting wall (where energy propagates obliquely rather than just in the $z$ direction).

We begin our analysis by assuming that all perturbations have an exp $(-i \omega t)$ time dependence so that Faraday's law becomes

$$
\nabla \times \tilde{\mathbf{E}}=i \omega \tilde{\mathbf{B}}
$$

and Ampère's law becomes

$$
\nabla \times \tilde{\mathbf{B}}=-i \frac{\omega}{c^{2}} \mathrm{~K} \cdot \tilde{\mathbf{E}}
$$

where $\mathrm{K}$ is given by Eq. (33). It is useful to normalize $\tilde{\mathbf{B}}$ to $c$ and spatial dimensions to the vacuum wave number $\omega / c$, i.e., we let

$$
\tilde{\mathbf{B}}_{\text {norm }}=c \tilde{\mathbf{B}}, \quad \mathbf{x}_{\text {norm }}=\frac{\omega}{c} \mathbf{x} ;
$$

however, for clarity we will omit writing the subscript 'norm'. Thus, using this normalization, Faraday's and Ampère's laws become simply

$$
\begin{aligned}
& \nabla \times \tilde{\mathbf{E}}=i \tilde{\mathbf{B}}, \\
& \nabla \times \tilde{\mathbf{B}}=-i \mathrm{~K} \cdot \tilde{\mathbf{E}} .
\end{aligned}
$$

Since the plasma is assumed uniform in the $z$ direction, we may Fourier analyze in $z$ and let all linearized quantities have the $z$ dependence $\exp \left(i n_{z} z\right)$. As before, $n_{z}$ is the refractive index in the $z$ direction, and is related to the parallel wave vector for unnormalized coordinates by $n_{z}=c k_{z} / \omega$.

We now split the perturbed fields and the $\nabla$ operator into components parallel and perpendicular to $z$, i.e.,

$$
\tilde{\mathbf{E}}=\tilde{\mathbf{E}}_{\perp}+\tilde{E}_{z} \hat{z}, \quad \tilde{\mathbf{B}}=\tilde{\mathbf{B}}_{\perp}+\tilde{B}_{z} \hat{z}, \quad \nabla=\nabla_{\perp}+i n_{z} \hat{z}
$$

and note that

$$
\mathrm{K} \cdot \tilde{\mathbf{E}}=S \tilde{\mathbf{E}}_{\perp}+i D \hat{z} \times \tilde{\mathbf{E}}_{\perp}+P \tilde{E}_{z} \hat{z} .
$$

Thus, Faraday and Ampère's laws can be written as

$$
\boldsymbol{\nabla}_{\perp} \times \tilde{\mathbf{E}}_{\perp}+\hat{z} \times\left(i n_{z} \tilde{\mathbf{E}}_{\perp}-\nabla_{\perp} \tilde{E}_{z}\right)=i\left(\tilde{\mathbf{B}}_{\perp}+\tilde{B}_{z} \hat{z}\right)
$$

and

$$
\begin{aligned}
\boldsymbol{\nabla}_{\perp} & \times \tilde{\mathbf{B}}_{\perp}+\hat{z} \times\left(i n_{z} \tilde{\mathbf{B}}_{\perp}-\nabla_{\perp} \tilde{B}_{z}\right) \\
& =-i\left(S \tilde{\mathbf{E}}_{\perp}+i D \hat{z} \times \tilde{\mathbf{E}}_{\perp}+P \tilde{E}_{z} \hat{z}\right) .
\end{aligned}
$$

Using the vector identity

$$
\hat{z} \cdot \nabla \times \mathbf{Q}=-\nabla \cdot(\hat{z} \times \mathbf{Q})=-\nabla_{\perp} \cdot(\hat{z} \times \mathbf{Q})
$$

the $z$ components of Eqs. (66) and (67) become

$$
\nabla_{\perp} \cdot\left(\hat{z} \times \tilde{\mathbf{E}}_{\perp}\right)=-i \tilde{B}_{z}
$$

and

$$
\boldsymbol{\nabla}_{\perp} \cdot\left(\hat{z} \times \tilde{\mathbf{B}}_{1}\right)=i P \tilde{E}_{z} .
$$


The perpendicular components of Eqs. (66) and (67) are

$$
\hat{z} \times\left(i n_{z} \tilde{\mathbf{E}}_{\perp}-\nabla_{\perp} \tilde{E}_{z}\right)=i \tilde{\mathbf{B}}_{\perp}
$$

and

$$
\hat{z} \times\left(i n_{z} \tilde{\mathbf{B}}_{\perp}-\nabla_{\perp} \tilde{B}_{z}\right)=-i\left(S \tilde{\mathbf{E}}_{\perp}+i D \hat{z} \times \tilde{\mathbf{E}}_{\perp}\right) .
$$

Equations (71) and (72) constitute two inhomogeneous linear equations in the four unknowns $\tilde{\mathbf{E}}_{\perp}, \tilde{\mathbf{B}}_{\perp}, \hat{z} \times \tilde{\mathbf{E}}_{\perp}$, and $\hat{z} \times \tilde{\mathbf{B}}_{\perp}$. Two additional independent equations in these four unknowns are obtained by crossing Eqs. (71) and (72) with $\hat{z}$, namely,

$$
-i n_{z} \tilde{\mathbf{E}}_{\perp}+\nabla_{\perp} \tilde{E}_{z}=i \hat{z} \times \tilde{\mathbf{B}}_{\perp}
$$

and

$$
-i n_{z} \tilde{\mathbf{B}}_{\perp}+\nabla_{\perp} \tilde{B}_{z}=-i \hat{z} \times S \tilde{\mathbf{E}}_{\perp}-D \tilde{\mathbf{E}}_{\perp} .
$$

Equations (71)-(74) can now be solved for the four unknowns to give
$\left[\begin{array}{c}\tilde{\mathbf{E}}_{\perp} \\ \overline{\mathbf{B}}_{\perp} \\ \hat{z} \times \tilde{\mathbf{E}}_{\perp} \\ \hat{z} \times \tilde{\mathbf{B}}_{\perp}\end{array}\right]=\frac{1}{\bar{s}^{2}-D^{2}}$

$$
\begin{gathered}
\times\left[\begin{array}{r}
i n_{z} \bar{S} \\
-n_{z}^{2} D \\
-n_{z} D \\
-i\left(S \bar{S}-D^{2}\right.
\end{array}\right. \\
\cdot\left[\begin{array}{c}
\nabla_{\perp} \tilde{E}_{z} \\
\nabla_{\perp} \tilde{B}_{z} \\
\hat{z} \times \nabla_{\perp} \tilde{E}_{z} \\
\hat{z} \times \nabla_{\perp} \tilde{B}_{z}
\end{array}\right]
\end{gathered}
$$

where again $\tilde{S}=S-n_{z}^{2}$. Thus, the fundamental field quantities are $\tilde{E}_{z}$ and $\tilde{B}_{z}$, since all other quantities can be calculated from these two quantities. The general cold plasma wave equation is obtained by substituting for $\hat{z} \times \tilde{\mathbf{E}}_{\perp}$ and $\hat{z} \times \tilde{\mathbf{B}}_{\perp}$ into Eqs. (69) and $(70)$ to obtain

$$
\nabla_{\perp} \cdot\left(\frac{\left(S \bar{S}-D^{2}\right) \nabla_{\perp} \tilde{E}_{z}-i n_{z} D \nabla_{\perp} \tilde{B}_{z}-i n_{z}^{2} D \hat{z} \times \nabla_{\perp} \tilde{E}_{z}-n_{z} \bar{S} \hat{z} \times \nabla_{\perp} \tilde{B}_{z}}{\bar{S}^{2}-D^{2}}\right)+P \tilde{E}_{z}=0
$$

and

$$
\begin{aligned}
& \nabla_{\perp} \cdot\left(\frac{i n_{z} D \nabla_{\perp} \tilde{E}_{z}+\tilde{S} \nabla_{\perp} \tilde{B}_{z}+n_{z} \bar{S} \hat{z} \times \nabla_{\perp} \tilde{E}_{z}-i D \hat{z} \times \nabla_{\perp} \tilde{B}_{z}}{\bar{S}^{2}-D^{2}}\right) \\
& \quad+\tilde{B}_{z}=0 .
\end{aligned}
$$

The denominator in these equations can be re-written as

$$
\bar{S}^{2}-D^{2}=\left(n_{z}^{2}-R\right)\left(n_{z}^{2}-L\right)
$$

so that its vanishing corresponds to the right and left handed circularly polarized modes propagating at $\theta=0$.

Also, since

$$
\nabla_{\perp} \cdot\left(\hat{z} \times \nabla_{\perp} \psi\right)=\nabla \cdot(\hat{z} \times \nabla \psi)=0
$$

all terms involving $\hat{z} \times \nabla_{\perp}$ may be simplified; for example,

$$
\nabla_{\perp} \cdot\left(\frac{i n_{z}^{2} D \hat{z} \times \nabla_{\perp} \tilde{E}_{z}}{\bar{S}^{2}-D^{2}}\right)=\left(\hat{z} \times \nabla_{\perp} \tilde{E}_{z}\right) \cdot \nabla_{\perp}\left(\frac{i n_{z}^{2} D}{\bar{S}^{2}-D^{2}}\right) \text {. }
$$

Using this simplification for all relevant terms, Eqs. (76) and (77) reduce to

$$
\begin{aligned}
& \nabla_{\perp} \cdot\left(\frac{\left(S \bar{S}-D^{2}\right)}{\bar{S}^{2}-D^{2}} \nabla_{\perp} \tilde{E}_{z}\right)+P \tilde{E}_{z} \\
& -\quad-i_{z}^{2}\left(\hat{z} \times \nabla_{\perp} \tilde{E}_{z}\right) \cdot \nabla_{\perp}\left(\frac{D}{\bar{S}^{2}-D^{2}}\right)
\end{aligned}
$$

$$
=\nabla_{\perp} \cdot\left(\frac{i n_{z} D}{\bar{S}^{2}-D^{2}} \nabla_{\perp} \tilde{B}_{z}\right)+n_{z}\left(\hat{z} \times \nabla_{\perp} \tilde{B}_{z}\right) \cdot \nabla_{\perp}\left(\frac{\bar{S}}{\bar{s}^{2}-D^{2}}\right)
$$

and

$$
\begin{aligned}
\boldsymbol{\nabla}_{\perp} & \cdot\left(\frac{\bar{S}}{\bar{S}^{2}-D^{2}} \nabla_{\perp} \tilde{B}_{z}\right)+\tilde{B}_{z}-i\left(\hat{z} \times \nabla_{\perp} \tilde{B}_{z}\right) \cdot \nabla_{\perp}\left(\frac{D}{\bar{S}^{2}-D^{2}}\right) \\
& =-\nabla_{\perp} \cdot\left(\frac{i n_{z} D}{\bar{S}^{2}-D^{2}} \nabla_{\perp} \tilde{E}_{z}\right)-\hat{z} \times \nabla_{\perp} \tilde{E}_{z} \cdot \nabla_{\perp}\left(\frac{n_{z^{\prime}}}{\bar{S}^{2}-D^{2}}\right) .
\end{aligned}
$$

Equations (81) and (82) provide an exact description (no approximations) of wave propagation in a cold inhomogeneous plasma for arbitrary $\omega$, arbitrary $n_{y}$ and arbitrary $n_{z}$; hence these equations provide a complete cold plasma description of shear and compressional Alfvén modes, ion cyclotron waves, lower hybrid waves, the 'fast' wave, upper hybrid waves, electron cyclotron waves, etc.

We will now discuss various limits of these general equations. Unless otherwise stated, slab geometry will be used and inhomogeneity, if any, will be assumed to be in the $x$ direction. Section V discusses situations where $n_{y}=0$, Sec. VI discusses situations where $n_{y}$ is finite, Sec. VII discusses 
a resonance cone behavior associated with the shear mode, and Sec. VIII discusses a shear mode toroidal current drive scheme.

\section{BEHAVIOR WHEN $N_{Y}=0$}

\section{A. Arbitrary frequency, inhomogeneous plasma,} $n_{y}=0$

Let us evaluate a typical term of Eqs. (81) or (82) involving the operator $\hat{z} \times \nabla_{\perp}$, e.g.,

$$
\left(\hat{z} \times \nabla_{\perp} \tilde{B}_{z}\right) \cdot \nabla_{\perp}\left(\frac{D}{\bar{S}^{2}-D^{2}}\right)=-i n_{y} \tilde{B}_{z} \frac{d}{d x}\left(\frac{D}{\bar{S}^{2}-D^{2}}\right) .
$$

Thus, if the plasma is homogeneous (i.e., $d / d x=0$ ) or if $n_{y}=0$, terms of this sort vanish.

We now assume that the plasma is inhomogeneous, but $n_{y}=0$. In this case Eqs. (81) and (82) reduce to

$$
\frac{d}{d x}\left(\frac{\left(S \bar{S}-D^{2}\right)}{\bar{S}^{2}-D^{2}} \frac{d \tilde{E}_{z}}{d x}\right)+P \tilde{E}_{z}=\frac{d}{d x}\left(\frac{i n_{z} D}{\bar{S}^{2}-D^{2}} \frac{d \tilde{B}_{z}}{d x}\right)
$$

and

$$
\frac{d}{d x}\left(\frac{\bar{S}}{\bar{S}^{2}-D^{2}} \frac{d \tilde{B}_{z}}{d x}\right)+\tilde{B}_{z}=-\frac{d}{d x}\left(\frac{\operatorname{in}_{z} D}{\bar{S}^{2}-D^{2}} \frac{d \tilde{E}_{z}}{d x}\right) .
$$

From Eqs. (84) and (85), it is easy to see the flaw in Eq. (44). The three steps effectively used in the argument leading to Eq. (44) were:

(1) since $|P| \rightarrow \infty$, Eq. (84) has the simple solution $\tilde{E}_{z} \rightarrow 0$ (this is essentially the basis of ideal MHD);

(2) since $\tilde{E}_{z} \rightarrow 0$, the right hand term of Eq. (85) is dropped, so that the two terms on the left hand side of Eq. (85) must balance each other;

(3) if we replace $d / d x$ by $i n_{x}$ then the left hand side (LHS) of Eq. (85) becomes Eq. (44), so that $n_{x}^{2} \rightarrow \infty$ when $\bar{S} \rightarrow 0$.

In actuality, this three-step argument fails when $\bar{S} \rightarrow 0$. What really happens when $\bar{S} \rightarrow 0$ is that the right hand side finite $\tilde{E}_{z}$ term of Eq. (85) balances the remaining term on the LHS, so that $n_{x}$ does not become infinite. It is not possible to set $\tilde{E}_{z}$ to zero while having $\tilde{B}_{z}$ finite (i.e., use the MHD assumption) since $\tilde{B}_{z}$ acts as a source term for $\tilde{E}_{z}$ in Eq. (84). Thus, the assumption that $\tilde{E}_{z}$ can be ignored is incorrect when $\bar{S} \rightarrow 0$. The Alfien resonance is thus a spurious artifact of ideal MHD resulting from the incorrect assumption that $\widetilde{E}_{z}$ is zero.

\section{B. Low frequency equations and dispersion relations}

If $\omega / \omega_{c i} \rightarrow 0$ then $D \rightarrow 0$ and also if $n_{y}=0$ then Eqs. (81) and (82) become two decoupled modes, an $E$ wave (Transverse Magnetic or TM mode) and a $B$ wave (Transverse Electric or TE mode)

$$
\nabla_{\perp} \cdot\left(\frac{S}{\bar{S}} \nabla_{\perp} \tilde{E}_{z}\right)+P \tilde{E}_{z}=0
$$

and

$$
\nabla_{\perp} \cdot\left(\frac{1}{\bar{S}} \nabla_{\perp} \tilde{B}_{z}\right)+\tilde{B}_{z}=0
$$

Because $\bar{S}$ occurs in the denominator of the coefficient of the highest order derivatives, the location $\bar{S}=0$ is a cutoff for both modes. Thus, the Alfven layer is a cutoff (place where $d / d x \rightarrow 0$ ) for both modes, not a resonance (place where $d / d x \rightarrow \infty)$. [Recall that in a uniform plasma the coordinate system can always be chosen to set $n_{y}=0$, so that Eqs. (86) and (87) are always valid for low frequency waves in a uniform plasma.]

We may Fourier analyze in the $x$ direction to obtain the dispersion relations

$$
n_{x}^{2}=\frac{P}{S}\left(S-n_{z}^{2}\right) \quad\{E \text { wave }\}
$$

and

$$
n_{x}^{2}=S-n_{z}^{2} \quad\{B \text { wave }\}
$$

\section{Identification of $E$ wave with the $n_{y}=0$ shear mode}

From Eq. (75) the low frequency perpendicular field components are found to be

$$
\begin{aligned}
& \tilde{\mathbf{E}}_{\perp}=\frac{1}{\bar{S}}\left(i n_{z} \nabla_{\perp} \tilde{E}_{z}-i \hat{z} \times \nabla_{\perp} \tilde{B}_{z}\right), \\
& \tilde{\mathbf{B}}_{\perp}=\frac{1}{\bar{S}}\left(i n_{z} \nabla_{\perp} \tilde{B}_{z}+i S \hat{z} \times \nabla_{\perp} \tilde{E}_{z}\right) .
\end{aligned}
$$

Thus, the field components which are finite for the $E$ wave when $n_{y}=0$ are

$$
\tilde{E}_{z} \neq 0, \quad \tilde{E}_{x}=\frac{i n_{z}}{\bar{S}} \frac{d \tilde{E}_{z}}{d x}, \quad \tilde{B}_{y}=\frac{i S}{\bar{S}} \frac{d \tilde{E}_{z}}{d x} .
$$

Comparison of Eq. (92) with Eq. (10) shows that the $E$ wave eigenvector corresponds to the shear wave [unboxed components in Eq. (10)]. We note that the dispersion given by Eq. (88) has been discussed in Ref, 40 where it was referred to as the Surface Alfvén Eigenmode (SAE).

Thus Eq. (88) is the correct form of the 'shear' Alfvén dispersion when $n_{x}$ is finite, and so we see that contrary to the prediction of ideal MHD, this 'shear' cold plasma mode does indeed depend on $n_{x}$. Although the $E$ wave discussed here involves the same field components as the MHD analysis of the shear wave, the spatial behavior is not the same. Consider the ratio $\tilde{E}_{x} / \tilde{B}_{y}$ predicted by Eq. (92) compared to that predicted by the normalized form of the third of Eqs. (11),

$$
\begin{aligned}
& \text { Eq. }(92) \Rightarrow \frac{\tilde{E}_{x}}{\tilde{B}_{y}}=\frac{n_{z}}{S}, \\
& \text { 3rd of Eqs. }(11) \Rightarrow \frac{\tilde{E}_{x}}{\tilde{B}_{y}}=\frac{1}{n_{z}} .
\end{aligned}
$$

These agree when $S=n_{z}^{2}$ but otherwise disagree. Thus, when $S \neq n_{z}^{2}$ there is a nontrivial difference between the predictions of ideal MHD and the predictions of our model. We 
therefore conclude, that ideal MHD gives an incorrect description of the shear Alfvén wave except at the Alfven layer.

\section{Why MHD fails to describe the $n_{y}=0$ shear mode except when $n_{z}^{2} \simeq S$}

\section{Error in y component of Faraday's law}

For simplicity consider the propagation of a pure $E$ wave in a uniform plasma. The third of Eqs. (11) is just the $y$ component of Faraday's law. How can there be anything wrong with an equation so basic to physics as Faraday's law? The answer is not that Faraday's law is wrong, but rather the way Faraday's law is being used is wrong. The problem with the third of Eqs. (11) is that it is based on the assumption that $\tilde{E}_{z}=0$. Consider the $y$ component of Faraday's law with $\tilde{E}_{z}$ retained, i.e.,

$$
n_{z} \tilde{E}_{x}-n_{x} \tilde{E}_{z}=\tilde{B}_{y}
$$

a box has been put around the term neglected in MHD. In the low frequency limit, the $x$ component of Ampère's law (essentially the $x$ component of the polarization current) gives another relation between $\tilde{E}_{x}$ and $\tilde{B}_{y}$ namely

$$
n_{z} \tilde{B}_{y}=S \tilde{E}_{x} \text {. }
$$

[Equation (95) comes from combining the first, second, and fourth of Eqs. (11) or equivalently from the ratio of the second and third of Eqs. (92).] Eliminating $\tilde{B}_{y}$ between Eqs. (94) and (95) gives

$$
n_{z} \tilde{E}_{x}-n_{x} \tilde{E}_{z}=\frac{S \tilde{E}_{x}}{n_{z}}
$$

so that the boxed term vanishes only when $n_{z}^{2}=S$; otherwise, it must be retained. When $n_{z}^{2} \gg S$ the right hand term is much smaller than the unboxed left hand term. Thus, the two left hand terms must nearly cancel, and in this case the wave is quasielectrostatic since the left side of Eq. (96) is the only nonzero component of $\nabla \times \tilde{\mathbf{E}}$ for $E$ wave polarization (the $x$ and $z$ components of $\boldsymbol{\nabla} \times \overline{\mathbf{E}}$ involve only $\tilde{E}_{y}$ which belongs to the $B$ wave polarization). To be more quantitative we may solve Eq. (96) for $n_{x} \tilde{E}_{z}$ giving

$$
n_{x} \tilde{E}_{z}=\left(n_{z}-\frac{S}{n_{z}}\right) \tilde{E}_{x}
$$

so the ratio of the boxed to the unboxed term on the left hand side is

$$
\frac{n_{x} \tilde{E}_{z}}{n_{z} \tilde{E}_{x}}=1-\frac{S}{n_{z}^{2}} .
$$

Thus, the two left hand terms nearly cancel when $n_{z}^{2} \gg S$, and the non-MHD term vanishes only when $S=n_{z}^{2}$. In summary, MHD gives an incorrect description of $E$ wave polarization when $n_{z}^{2} \gg S$, because MHD omits the $n_{x} \tilde{E}_{z}$ term that nearly cancels the $n_{z} \tilde{E}_{x}$ term assumed to be dominant in MHD.

\section{Dispersion relation point of view}

Another way of viewing this problem is to look at the first and third lines of Eq. (36) for low frequencies (i.e., $D \rightarrow 0$ ); these give two coupled equations in $\tilde{E}_{x}$ and $\tilde{E}_{z}$,

$$
\begin{aligned}
& \left(S-n_{z}^{2}\right) \tilde{E}_{x}+n_{x} n_{z} \tilde{E}_{z}=0, \\
& n_{x} n_{z} \tilde{E}_{x}+\left(P-n_{x}^{2}\right) \tilde{E}_{z}=0 .
\end{aligned}
$$

The traditional MHD argument (e.g., Ref. 41) is to say (i) because the frequency is low, $P \rightarrow \infty$; (ii) because the coefficient of $\tilde{E}_{z}$ in Eq. (100) becomes infinite, $\tilde{E}_{z}$ must be approximately zero; (iii) therefore $\tilde{E}_{z}$ can be neglected in Eq. (99) which then becomes the MHD shear wave dispersion $n_{z}^{2}=S$, i.e., $\omega^{2}=k_{z}^{2} v_{A}^{2}$. This traditional argument is not correct, because although $\tilde{E}_{z}$ may be small, $n_{x}$ is typically large so that the product $n_{x} \tilde{E}_{z}$ is not negligible. Solving for $\tilde{E}_{z}$ using Eq. (100) and substituting in Eq. (99) gives

$$
S-n_{z}^{2}-n_{x}^{2} n_{z}^{2} /\left(P-n_{x}^{2}\right)=0
$$

where we have put a box around the terms resulting from finite $\tilde{E}_{z}$. Cross-multiplying gives

$$
\left(S-n_{z}^{2}\right)\left(P-n_{x}^{2}\right)-n_{x}^{2} n_{z}^{2}=0
$$

or

$$
S P-n_{x}^{2} S-n_{z}^{2} P+n_{x}^{2} n_{z}^{2}-n_{x}^{2} n_{z}^{2}=0 .
$$

The MHD and non-MHD $n_{x}^{2} n_{z}^{2}$ terms (boxed and unboxed) cancel and so one obtains Eq. (88), which shows that $n_{x}^{2}$ is indeed large except when $n_{z}^{2} \simeq S$. We have shown the algebraic detail to emphasize that it is not correct to ignore the $\tilde{E}_{z}$ term (boxed term).

Also, if we look again at Eq. (100) we again see the violation of quasineutrality discussed in Sec. III G; arbitrarily setting $\tilde{E}_{z}=0$ forces $n_{x} \tilde{E}_{x}=0$, but this is inconsistent with the MHD assumption that both $n_{x}$ and $\tilde{E}_{x}$ are finite.

\section{E. Why the shear mode is quasielectrostatic except when $n_{z}^{2} \simeq S$}

In general, a wave electric field $\tilde{\mathbf{E}}$ can be split into a longitudinal component $\tilde{\mathbf{E}}^{l}$ and a transverse component $\tilde{\mathbf{E}}^{t}$ relative to the refractive index vector $\mathbf{n}$, i.e.,

$$
\tilde{\mathbf{E}}=\tilde{\mathbf{E}}^{l}+\tilde{\mathbf{E}}^{t}
$$

where

$$
\tilde{\mathbf{E}}^{l}=\hat{n} \hat{n} \cdot \tilde{\mathbf{E}}, \quad \tilde{\mathbf{E}}^{t}=\hat{n} \times(\tilde{\mathbf{E}} \times \hat{n})
$$

and $\hat{n}=\mathbf{n} / n$ is the refractive index unit vector. Using this definition we see that $\boldsymbol{\nabla} \cdot \tilde{\mathbf{E}}^{t}=i \mathbf{n} \cdot \tilde{\mathbf{E}}^{t}=0$ and $\boldsymbol{\nabla} \times \tilde{\mathbf{E}}^{l}$ $=i \mathbf{n} \times \tilde{\mathbf{E}}^{l}=0$ so that the longitudinal component corresponds to the electrostatic electric field (i.e., the electric field produced by net charge density), and the transverse component corresponds to the inductive electric field (i.e., the electric field produced by time-changing magnetic fields).

The extent to which a mode is electrostatic can be determined by calculating the ratio of the clectrostatic electric field to the inductive electric field, i.e.,

$$
\left|\frac{\tilde{\mathbf{E}}^{\prime}}{\tilde{\mathbf{E}}^{t}}\right|=\left|\frac{\mathbf{n} \cdot \tilde{\mathbf{E}}}{\mathbf{n} \times \tilde{\mathbf{E}}}\right|=\left|\frac{n_{x} \tilde{E}_{x}+n_{z} \dot{E}_{z}}{n_{x} \tilde{E}_{z}-n_{z} \tilde{E}_{x}}\right| .
$$

From Eq. (97) it is seen that

$$
\tilde{E}_{x}=-\frac{n_{x} n_{z}}{\bar{S}} \tilde{E}_{z}
$$

so that Eq. (106) becomes 


$$
\left|\frac{\tilde{\mathbf{E}}^{\prime}}{\tilde{\mathbf{E}}^{\prime}}\right|=\left|\frac{n_{z}}{n_{x}}\left(\frac{n_{x}^{2} / \bar{S}-1}{n_{z}^{2} / \bar{S}+1}\right)\right|
$$

or using the dispersion relation, Eq. (88), to eliminate $n_{x}$,

$$
\left|\frac{\tilde{\mathbf{E}}^{\imath}}{\tilde{\mathbf{E}}^{t}}\right|-\frac{n_{z}}{S} \sqrt{\frac{|P \bar{S}|}{S}}\left|1-\frac{S}{P}\right| \simeq \frac{n_{z}}{\sqrt{S}} \frac{\omega_{g m}}{\omega} \sqrt{\frac{\bar{S}}{S}}
$$

where we have used $|1-S / P| \simeq 1$ since $|P / S| \gg 1$. Let us write $S=n_{z}^{2}+\delta S$ so that $\delta S=\bar{S}$ is a measure of the deviation of $S$ from its value at the Alfvén layer. At the Alfvén layer, $\bar{S}=0$ so from Eq. (109) the wave is purely inductive (transverse). However, at a slight distance away from the Alfvén layer, Eq. (109) becomes

$$
\left|\frac{\tilde{\mathbf{E}}^{\prime}}{\tilde{\mathbf{E}}^{t}}\right|=\frac{\omega_{g m}}{\omega} \sqrt{\frac{\delta S}{S}} .
$$

Thus, the electrostatic component becomes equal in magnitude to the inductive component when

$$
\frac{\delta S}{S}=\frac{\omega^{2}}{\omega_{g m}^{2}}=\frac{m_{e}}{m_{i}} \frac{\omega^{2}}{\omega_{c i}^{2}} .
$$

Since, by assumption $\omega \ll \omega_{c i}$ it is clear that the wave becomes dominantly electrostatic within an extremely short distance from the Alfvén layer. Thus, the shear wave is electrostatic except in the immediate vicinity of the Alfven layer. Since MHD cannot describe electrostatic behavior, the MHD description of the $n_{y}=0$ shear wave can only be valid in the immediate vicinity of the Alfvén layer; elsewhere the MHD description of the cold plasma $n_{y}=0$ shear wave is invalid.

\section{F. Identification of $B$ wave with the compressional mode}

Now consider Eq. (89), the $B$ wave. From Eqs. (90) and (91) the nonvanishing field components associated with this mode are

$$
\tilde{B}_{z} \neq 0, \quad \tilde{E}_{y}=-\frac{i}{\bar{S}} \frac{d \tilde{B}_{z}}{d x}, \quad \tilde{B}_{x}=\frac{i n_{z}}{\bar{S}} \frac{d \tilde{B}_{z}}{d x}
$$

Comparison of Eq. (112) with Eq. (10) shows that the $B$ wave cigenvector corresponds to the compressional wave [boxed components in Eq. (10)]. Thus, we may identify the $B$ wave as being the compressional mode.

Consider the ratio $\tilde{E}_{y} / B_{x}$ predicted by Eq. (112) and by the normalized form of the fourth of Eqs. (13),

Eq. (112) $\Rightarrow \frac{\tilde{E}_{y}}{\tilde{B}_{x}}=-\frac{1}{n_{z}}$

4th of Eqs. (13) $\Rightarrow \frac{\tilde{E}_{y}}{\tilde{B}_{x}}=-\frac{1}{n_{z}}$.

We see that here there is agreement between our model and the fourth of Eqs. (13) which is the $x$ component of Faraday's law and conclude that the MHD description of the compressional mode is generally correct. Thus, ideal MHD may be used for $n_{y}=0$ compressional waves, but gives an incorrect description of $n_{y}=0$ shear waves except at the Al- fvén layer. The electric field for the compressional wave is always purely inductive, consistent with the conclusion that MHD provides a valid description for the $n_{y}=0$ compressional mode.

From the dispersion relations, we see that the layer where $S=n_{z}^{2}$ [i.e., where $\omega=k_{z} v_{A}(x)$ for $v_{A} \ll c$ ] is a cutoff for both the $E$ and $B$ waves. Since $P<0$ for low frequency cold plasma waves, the shear mode propagates when $n_{z}^{2}>S$ (i.e., on the low density, high field side of the cutoff), while the compressional mode propagates on the opposite side.

\section{G. Circumstances when the MHD description of shear Alfvén waves is valid}

We have seen that the shear Alfvén wave which has dispersion equation (88) is quasielectrostatic except in the close vicinity of the Alfvén layer and so in general cannot be described by the MHD equations. The way Eq. (88) is used depends on the way boundary conditions are imposed. If the excitation is from the side, then $n_{z}$ is prescribed by the antenna geometry and the dispersion relation determines $n_{x}$. In this case, because $P / S$ is very large, the dispersion relation gives a large $n_{x}$, except at the Alfven layer where $S-n_{z}^{2} \simeq 0$. On the other hand, if the excitation is from the end (which is the case for all experiments that have demonstrated the dispersion $\omega=k_{z} v_{A}$ ), then $n_{x}$ is prescribed by the antenna and so Eq. (88) determines $n_{z}$. Now, the leverage is reversed so that unless $n_{x}$ is very large, Eq. (88) forces $n_{z}^{2}$ to be approximately equal to $S$.

In summary, the simple MHD model of shear Alfvén waves is appropriate for end excitation if a small $n_{x}$ is prescribed, but MHD is generally inappropriate for side excitation since a modest $n_{z}$ will give a large $n_{x}$ at all regions other than the Alfvén layer.

\section{BEHAVIOR WHEN $N_{Y}$ IS FINITE}

\section{A. Finite $n_{y}$ low frequency limit}

In the low frequency limit $D \rightarrow 0$, and so Eqs. (81) and (82) assume the form

$$
\nabla_{\perp} \cdot\left(\frac{S}{\bar{S}} \nabla_{\perp} \tilde{E}_{z}\right)+P \tilde{E}_{z}=n_{z}\left(\hat{z} \times \nabla_{\perp} \tilde{B}_{z}\right) \cdot \nabla_{\perp}\left(\frac{1}{\bar{S}}\right)
$$

and

$$
\nabla_{\perp} \cdot\left(\frac{1}{\bar{S}} \nabla_{\perp} \tilde{B}_{z}\right)+\tilde{B}_{z}=-n_{z} \hat{z} \times \nabla_{\perp} \tilde{E}_{z} \cdot \nabla_{\perp}\left(\frac{1}{\bar{S}}\right) .
$$

We now assert that (i) Eq. (115) corresponds to Eq. (32), except that Eq. (32) is missing the right hand side of Eq. (115), and (ii) all fields are finite in the vicinity of $\bar{S}=0$ even when $n_{y}$ is finite.

Let us start with Eq. (115), but omit the right hand side (which of course is not correct if $n_{y}$ is finite), i.e., we start with

$$
\begin{aligned}
\nabla_{\perp} \cdot\left(\frac{1}{S-n_{z}^{2}} \nabla_{\perp} \tilde{B}_{z}\right)+\tilde{B}_{z}=0 \\
\\
\left\{\tilde{E}_{z} \text { incorrectly forced to zero }\right\}
\end{aligned}
$$


or

$$
\begin{aligned}
& \frac{d}{d x}\left(\frac{1}{S-n_{z}^{2}} \frac{d}{d x} \tilde{B}_{z}\right)-\frac{n_{y}^{2}}{S-n_{z}^{2}} \tilde{B}_{z}+\tilde{B}_{z}=0 \\
& \left\{\tilde{E}_{z} \text { incorrectly forced to zero }\right\} .
\end{aligned}
$$

To prevent inadvertent use of these equations in other contexts, we have explicitly noted that $\tilde{E}_{z}$ has been incorrectly forced to be zero.

From Eq. (75)

$$
\begin{aligned}
\tilde{\mathbf{E}}_{\perp}= & \frac{1}{\bar{S}^{2}-D^{2}}\left(i n_{z} \bar{S} \nabla_{\perp} \tilde{E}_{z}+D \nabla_{\perp} \tilde{B}_{z}+n_{z} D \hat{z} \times \nabla_{\perp} \tilde{E}_{z}\right. \\
& \left.-i \bar{S} \hat{z} \times \nabla_{\perp} \tilde{B}_{z}\right)
\end{aligned}
$$

so that in the limit $D \rightarrow 0$ and again setting $\tilde{E}_{z}$ artificially to zero, we obtain

$$
\tilde{\mathbf{E}}_{\perp}=-\frac{i}{\bar{S}} \hat{z} \times \nabla_{\perp} \tilde{B}_{z} \quad\left\{\tilde{E}_{z} \text { incorrectly forced to zero }\right\}
$$

so that

$$
i \tilde{E}_{y}=\frac{1}{S-n_{z}^{2}} \frac{d \tilde{B}_{z}}{d x} \quad\left\{\tilde{E}_{z} \text { incorrectly forced to zero }\right\} .
$$

Thus, Eq. (117) becomes, using Eq. (120),

$$
\begin{aligned}
i \frac{d \tilde{E}_{y}}{d x}+\left[\frac{S-\left(n_{y}^{2}+n_{z}^{2}\right)}{S-n_{z}^{2}}\right] \tilde{B}_{z}=0 \\
\left\{\tilde{E}_{z} \text { incorrectly forced to zero }\right\}
\end{aligned}
$$

or, solving for $\tilde{B}_{z}$,

$$
\tilde{B}_{z}=\frac{-i\left(S-n_{z}^{2}\right)}{S-\left(n_{y}^{2}+n_{z}^{2}\right)} \frac{d \tilde{E}_{y}}{d x} .
$$

Inserting Eq. (122) in Eq. (120) gives

$$
\frac{d}{d x}\left[\frac{\left(S-n_{z}^{2}\right)}{S-\left(n_{y}^{2}+n_{z}^{2}\right)} \frac{d \tilde{E}_{y}}{d x}\right]+\left(S-n_{z}^{2}\right) \tilde{E}_{y}=0 .
$$

If we note that $S=1+c^{2} / v_{A}^{2}$ and use the MHD assumption that $v_{A} \ll c$, this becomes

$$
\frac{d}{d x}\left[\frac{\left(c^{2} / v_{A}^{2}-n_{z}^{2}\right)}{c^{2} / v_{A}^{2}-\left(n_{y}^{2}+n_{z}^{2}\right)} \frac{d \tilde{E}_{y}}{d x}\right]+\left(c^{2} / v_{A}^{2}-n_{z}^{2}\right) \tilde{E}_{y}=0
$$

or, in unnormalized quantities

$$
\frac{d}{d x}\left[\frac{\left(\omega^{2} / v_{A}^{2}-k_{z}^{2}\right)}{\omega^{2} / v_{A}^{2}-\left(k_{y}^{2}+k_{z}^{2}\right)} \frac{d \tilde{E}_{y}}{d x}\right]+\left(\omega^{2} / v_{A}^{2}-k_{z}^{2}\right) \tilde{E}_{y}=0 .
$$

From the MHD Ohm's law, Eq. (2), the $x$ component of the MHD velocity is

$$
\tilde{U}_{x}=\frac{\tilde{E}_{y}}{B}
$$

so we see that Eq. (125) is mathematically equivalent to Eq. (32).
Thus, Eq. (32) describes only the compressional Alfvén wave and, contrary to the assertions in Refs. 3 and 4, does not describe the shear wave. The essential point here is that Eq. (32) is missing the finite $\tilde{E}_{z}$ terms which are critically important in the region where $S \approx n_{z}^{2}$ and which prevent the equations from becoming singular.

\section{B. Mode coupling in an inhomogeneous plasma when $n_{y} \neq 0$}

References 3 and 4 claimed that Eq. (32) has a logarithmic singularity in the vicinity of the $\omega=k_{z} v_{A}$ layer. In the previous section we showed that Eq. (32) is incorrect because it is missing terms involving $\tilde{E}_{z}$. In this section we show that when these terms are properly taken into account, there is no singularity at the $\omega=k_{z} v_{A}$ layer and that, instead, there is a strong coupling between $\tilde{E}_{z}$ and $\tilde{B}_{z}$.

We consider the low frequency limit (i.e., $D \rightarrow 0$ ) but allow both plasma inhomogeneity in the $x$ direction and finite $n_{y}$. Thus, Eqs. (114) and (115) become

$$
\begin{aligned}
& \frac{d}{d x}\left(\frac{S}{S-n_{z}^{2}} \frac{d \tilde{E}_{z}}{d x}\right)-\frac{n_{y}^{2} S}{S-n_{z}^{2}} \tilde{E}_{z}+P \tilde{E}_{z} \\
& \quad=-i n_{y} n_{z} \tilde{B}_{z} \frac{d}{d x}\left(\frac{1}{S-n_{z}^{2}}\right)
\end{aligned}
$$

and

$$
\begin{aligned}
& \frac{d}{d x}\left(\frac{1}{S-n_{z}^{2}} \frac{d \tilde{B}_{z}}{d x}\right)-\frac{n_{y}^{2}}{S-n_{z}^{2}} \tilde{B}_{z}+\tilde{B}_{z} \\
& \quad=i n_{y} n_{z} \tilde{E}_{z} \frac{d}{d x}\left(\frac{1}{S-n_{z}^{2}}\right) .
\end{aligned}
$$

Note that the left hand side of Eq. (128) is equivalent to Eq. (32). We define the $x$ origin to be where $S=n_{z}^{2}$, so in the vicinity of the origin we can write

$$
S-n_{z}^{2}=x S^{\prime}
$$

where the prime means $d / d x$. Thus, in the vicinity of $x=0$, Eqs. (127) and (128) become

$\frac{d}{d x}\left(\frac{n_{z}^{2}}{x S^{\prime}} \frac{d \tilde{E}_{z}}{d x}\right)-\frac{n_{y}^{2} n_{z}^{2}}{x S^{\prime}} \tilde{E}_{z}+P \tilde{E}_{z}=-i n_{y} n_{z} \tilde{B}_{z} \frac{d}{d x}\left(\frac{1}{x S^{\prime}}\right)$

and

$$
\frac{d}{d x}\left(\frac{1}{x S^{\prime}} \frac{d \tilde{B}_{z}}{d x}\right)-\frac{n_{y}^{2}}{x S^{\prime}} \tilde{B}_{z}+\tilde{B}_{z}=i n_{y} n_{z} \tilde{E}_{z} \frac{d}{d x}\left(\frac{1}{x S^{\prime}}\right),
$$

or, after some simplification and the dropping of terms that do not contain inverse powers of $x$,

$$
\frac{d}{d x}\left(\frac{1}{x} \frac{d\left(n_{z} \tilde{E}_{z}\right)}{d x}\right)-\frac{n_{y}^{2}}{x}\left(n_{z} \tilde{E}_{z}\right)=\frac{i n_{y} \tilde{B}_{z}}{x^{2}}
$$

and

$$
\frac{d}{d x}\left(\frac{1}{x} \frac{d \tilde{B}_{z}}{d x}\right)-\frac{n_{y}^{2}}{x} \tilde{B}_{z}=-\frac{i n_{y}\left(n_{z} \tilde{E}_{z}\right)}{x^{2}} .
$$

We now define

$$
Q_{ \pm}=n_{z} \tilde{E}_{z} \pm i \tilde{B}_{z}
$$


and add Eq. (131) to $\pm i$ times Eq. (132) obtaining

$$
\frac{d}{d x}\left(\frac{1}{x} \frac{d Q_{ \pm}}{d x}\right)-\frac{n_{y}^{2}}{x} Q_{ \pm}=\frac{ \pm n_{y} Q_{ \pm}}{x^{2}}
$$

or

$$
\frac{d^{2} Q_{ \pm}}{d x^{2}}-\frac{1}{x} \frac{d Q_{ \pm}}{d x}-\left(n_{y}^{2} \pm \frac{n_{y}}{x}\right) Q_{ \pm}=0 .
$$

Equation (135) can be transformed ${ }^{42}$ to a standard form by defining

$$
\xi=2 \sqrt{n_{y} x}
$$

so that

$$
x^{1 / 2}=\frac{\xi}{2 \sqrt{n_{y}}}, \quad \frac{d \xi}{d x}=\frac{2 n_{y}}{\xi}
$$

and let

$$
Q_{ \pm}=\frac{\xi^{2}}{4} g_{ \pm}(\xi)
$$

Thus,

$$
\frac{d Q_{ \pm}}{d x}=n_{y} g_{ \pm}+\frac{n_{y}}{2} \xi_{ \pm}^{\prime}
$$

and

$$
\frac{d^{2} Q_{ \pm}}{d x^{2}}=\frac{3 n_{y}^{2}}{\xi} g_{ \pm}^{\prime}+n_{y}^{2} g_{ \pm}^{\prime \prime}
$$

so that Eq. (135) becomes in the limit of small $\xi$

$$
\xi^{2} g_{ \pm}^{\prime \prime}+\xi g_{ \pm}^{\prime}-\left(4 \pm \xi^{2}\right) g_{ \pm}(\xi)=0 \text {. }
$$

Equation (141) is a Bessel's equation of order 2 when the minus sign is chosen and is a modified Bessel's equation of order 2 when the plus sign is chosen. Thus, for small $\xi$ we find

$$
Q_{-}=\frac{\xi^{2}}{4}\left[a J_{2}(\xi)+b Y_{2}(\xi)\right]
$$

and

$$
Q_{+}=\frac{\xi^{2}}{4}\left[\alpha I_{2}(\xi)+\beta K_{2}(\xi)\right]
$$

where $a, b, \alpha$, and $\beta$ are constants which will be determined by the boundary conditions at large $\xi$. The important point here is that for small $\xi$

$$
J_{2}(\xi), I_{2}(\xi) \sim \xi^{2}
$$

while

$$
Y_{2}(\xi), K_{2}(\xi)-\xi^{-2}
$$

so that both $Q_{-}$and $Q_{+}$are finite and well behaved at $\xi=0$ (i.e., $\tilde{E}_{z}$ and $\tilde{B}_{z}$ are finite and well behaved at $\omega=k_{z} v_{A}$ ). Thus, even for finite $n_{y}$ there is no Alfvén resonance (i.e., no singularity at $x=0$ ); hence, there should not be any spccial absorption at $x=0$. However, we note that finite $n_{y}$ causes a strong coupling of the shear and compressional modes in the vicinity of $x=0$; this linear mode coupling in the presence of inhomogeneity in the $x$ direction and finite $n_{y}$ is an important phenomenon completely overlooked by conventional uniform cold plasma theory. Standard asymptotic formulas will connect these inner, small $|x|$ solutions to the outer, large $|x|$ Wentzel-Kramer-Brillouin (WKB) solutions. These connecting formulas will be complicated and are beyond the scope of the present paper, but will not introduce any singularities since the WKB solutions are nonsingular and well behaved in the regions away from $x=0$. The only possible place for a singularity was at $x=0$ and we have demonstrated that the solution is well behaved and finite at this point. Thus, we have disproved the assertion in Refs. 3 and 4 that a compressible cold plasma has a logarithmic singularity at the $\omega=k_{z} v_{A}$ layer when $n_{y}$ is finite.

\section{Why the Alfvén resonance concept also fails for perpendicular field components of finite $n_{y}$ modes}

References $2,3,9,13$ and many following papers argue that the cold plasma shear Alfvén wave has a logarithmic singularity at the Alfvén layer. This singularity is manifested by the MHD velocity $\tilde{U}_{x}$ which, as shown in Eq. (126) is proportional to $\tilde{E}_{y}$. Furthermore, Refs. 2, 5, and 9 argue that a spatial phase mixing occurs at the Alfvén resonance, producing strong wave absorption and plasma heating at this layer; the elaborate mathematical model used to derive the phase mixing was summarized in Sec. II F. Reference 5 argues that the magnitude of the absorption can be calculated from the ideal MHD equations without actually knowing the details of the absorption mechanism. We now demonstrate that the logarithmically singular Alfvén "resonance", the associated phase mixing and absorption are all nonexistent.

In the vicinity of the Alfvén layer, i.e, where $S \approx n_{z}^{2}$, Eqs. (127) and (128) have the limiting form

$$
\frac{d\left(n_{z} \tilde{E}_{z}\right)}{d x}=-i n_{y} \tilde{B}_{z}
$$

and

$$
\frac{d \tilde{B}_{z}}{d x}=i n_{y}\left(n_{z} \tilde{E}_{z}\right)
$$

where we have used the result from Sec. VI B that both $E_{z}$ and $B_{z}$ are finite and well behaved at the Alfvén layer, and we have kept only the dominant terms in Eqs. (127) and (128), i.e., those terms scaling as $\left(S-n_{z}^{2}\right)^{-2}$.

Let us examine $\tilde{E}_{x}$ and $\tilde{E}_{y}$; using Eq. (90) these are found to be

$$
\tilde{E}_{x}=\frac{\left[i n_{z}\left(d \tilde{E}_{z} / d x\right)-n_{y} \tilde{B}_{z}\right]}{S-n_{z}^{2}}
$$

and

$$
\tilde{E}_{y}=-\frac{\left[n_{z} n_{y} \tilde{E}_{z}+i\left(d \tilde{B}_{z} / d x\right)\right]}{S-n_{z}^{2}} .
$$

If, as in ideal MHD, $\tilde{E}_{z}$ is arbitrarily and unjustifiably forced to be zero, then Eqs. (148) and (149) indeed become singular at the Alfvén layer where $S=n_{z}^{2}$ [this is the essence of the claimed ideal MHD Alfvén resonance resulting from 
Eq. (32)]. However, if $\tilde{E}_{z}$ is properly taken into account, then we see from Eqs. (146) and (147) that the leading order $\tilde{E}_{z}$ terms in the numerators of Eqs. (148) and (149) cancel the leading order $\tilde{B}_{z}$ terms so that the numerator goes to zero in the same fashion as the denominator. Similar behavior occurs for $\tilde{B}_{x}$ and $\tilde{B}_{y}$ in the vicinity of the Alfvén layer. Thus, even when $n_{y}$ is finite there is still no singularity at the Alfven layer; all six components of $\tilde{\mathbf{E}}$ and $\mathbf{B}$ are finite and well behaved at the Alfven layer as is $\tilde{\mathbf{U}}$. The fact that ideal MHD predicted a spurious resonance with associated absorption constitutes a serious failure of the ideal MHD model of low frequency plasma behavior.

It is often argued that, despite its shortcomings, ideal MHD is a 'robust model' of the plasma and provides a good description of plasma behavior even in regimes when it should not be valid, and that in any case, shortcomings can be patched up in ad hoc fashion. This argument is clearly inappropriate here. Ideal MHD is not a robust model of the ideal cold plasma here because ideal MHD predicted a nonexistent singularity and a nonexistent heating mechanism. This is an error that cannot be 'patched up'.

\section{Numerical solutions of coupled wave equations}

If there were a singularity at the Alfvén layer, it would not be possible to integrate the wave equations across the layer without introducing a dissipation. To emphasize that there is no singularity, we present numerical integrations of Eqs. (114) and (115) and show that these solutions are well behaved at the Alfvén layer-no dissipation needs to be introduced to resolve singularities. In order to relate to experiments we will use cylindrical geometry (the numerical integration could also be done in slab geometry).

In cylindrical geometry Eqs. (114) and (115) become

$$
\frac{1}{r} \frac{d}{d r}\left[\frac{r S}{\bar{S}} \frac{d \psi}{d r}\right]+\left[-\frac{m^{2} S}{r^{2} \bar{S}}+P\right] \psi=-\frac{m n_{z}}{r} \chi \frac{d}{d r}\left(\frac{1}{\bar{S}}\right)
$$

and

$$
\frac{1}{r} \frac{d}{d r}\left[\frac{r}{\bar{S}} \frac{d \chi}{d r}\right]+\left[-\frac{m^{2}}{r^{2} \bar{S}}+1\right] \chi=-\frac{m n_{z}}{r} \psi \frac{d}{d r}\left(\frac{1}{\bar{S}}\right)
$$

where $\psi=\tilde{E}_{z}$ and $\chi=i \tilde{B}_{z}$. We rearrange these equations into the form

$$
f_{1} \psi^{\prime \prime}+g_{1} \psi^{\prime}+h_{1} \psi=\lambda \chi, \quad f_{2} \chi^{\prime \prime}+g_{2} \chi^{\prime}+h_{2} \chi=\lambda \psi
$$

where

$f_{1}=S \bar{S}, \quad g_{1}=\frac{S \bar{S}}{r}-n_{z}^{2} \frac{d S}{d r}, \quad h_{1}=-\frac{m^{2}}{r^{2}} S \bar{S}+P \bar{S}^{2}$,

$f_{2}=\bar{S}, \quad g_{2}=\frac{\bar{S}}{r}-\frac{d S}{d r}, \quad h_{2}=-\frac{m^{2}}{r^{2}} \bar{S}+\bar{S}^{2}, \quad \lambda=\frac{m n_{z}}{r} \frac{d S}{d r}$.

We choose two cases, the first approximating the parameters of the Tokapole II experiment ${ }^{28}$ and the second the parameters of a reactor-grade tokamak. The Tokapole II experiment had $m=1$ symmetry so we will use $m=1$.
Equation (152) is solved in a single iteration using a coupled tridiagonal matrix inversion scheme over a 1000 point grid [these numerical solutions are very accurate; when substituted back into the original difference equation form of Eqs. (152), the numerical solutions satisfy the difference equations within the roundoff error of the computer]. All that is required for inputs are boundary conditions for $\psi$ and $\chi$ on axis (i.e., at $r=0$ ) and at the edge $r=a$. Because of the $m=1$ symmetry, regularity conditions at $r=0$ require ${ }^{43}$ that $\psi(0)=\chi(0)=0$ always. Thus, $E$ wave excitation is described by using edge boundary conditions $\psi(a)=1, \chi(a)=0$, while $B$ wave excitation is described by using edge boundary conditions $\psi(a)=0, \chi(a)=1$.

Figure 2 shows results of the numerical computation for parameters similar to the Tokapole II experiment while Fig. 3 shows results for parameters of a large tokamak. It should be noted that in the limit that the plasma density goes to zero Eqs. (150) and (151) transform smoothly and continuously to the vacuum TE and TM cylindrical waveguide modes; this is easily demonstrated by letting $S \rightarrow 1, P \rightarrow 1$. At very low densities, the quasielectrostatic $E$ wave exists over the entire plasma radius, but as the density is increased, the domain of this mode is pushed radially outward (to the right of the Alfvén layer). The much longer wavelength $B$ wave becomes evident at higher densities. From Fig. 2 it is seen that as the

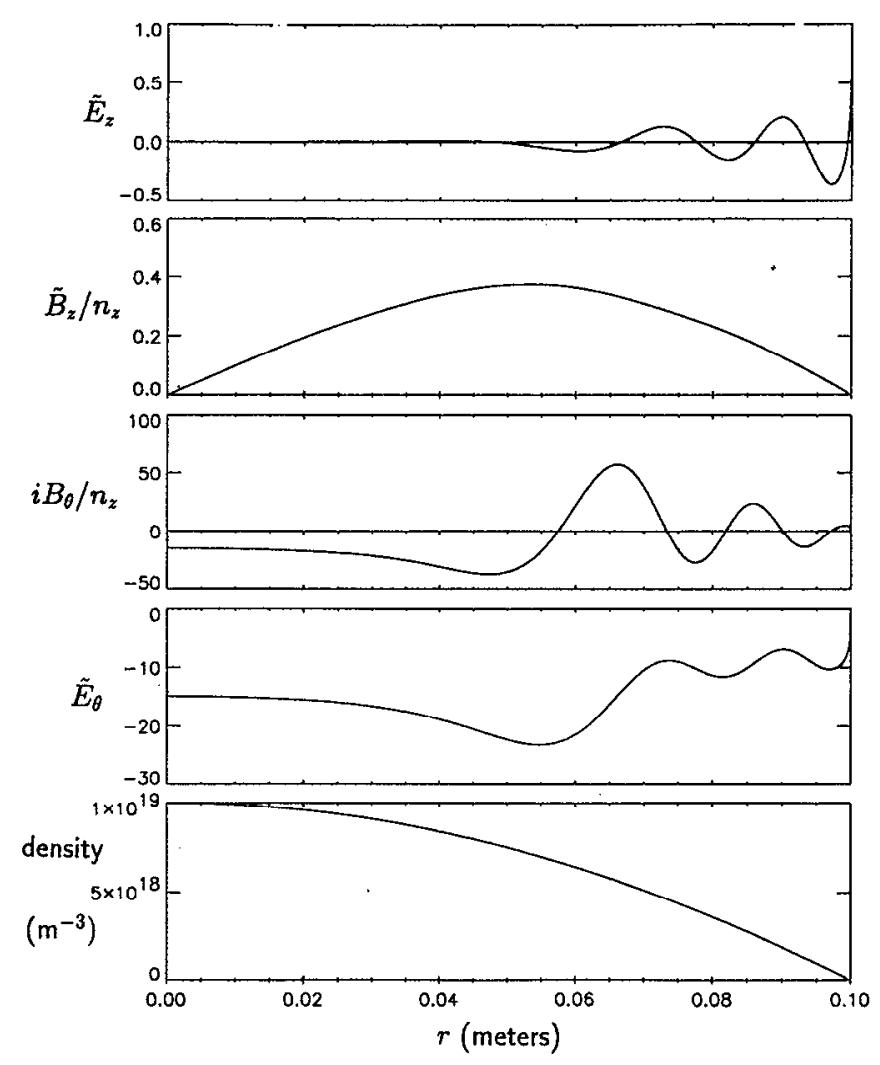

FIG. 2. Numerical solution of Eqs. (150), (151) and associated perpendicular components for parameters similar to Tokapole $\Pi$ experiment, ${ }^{28}$ hydrogen plasma, $f=1.16 \mathrm{MHz}, B=0.5 \mathrm{~T}, n_{z}=74, m=1, \tilde{E}_{z}$ excitation. 


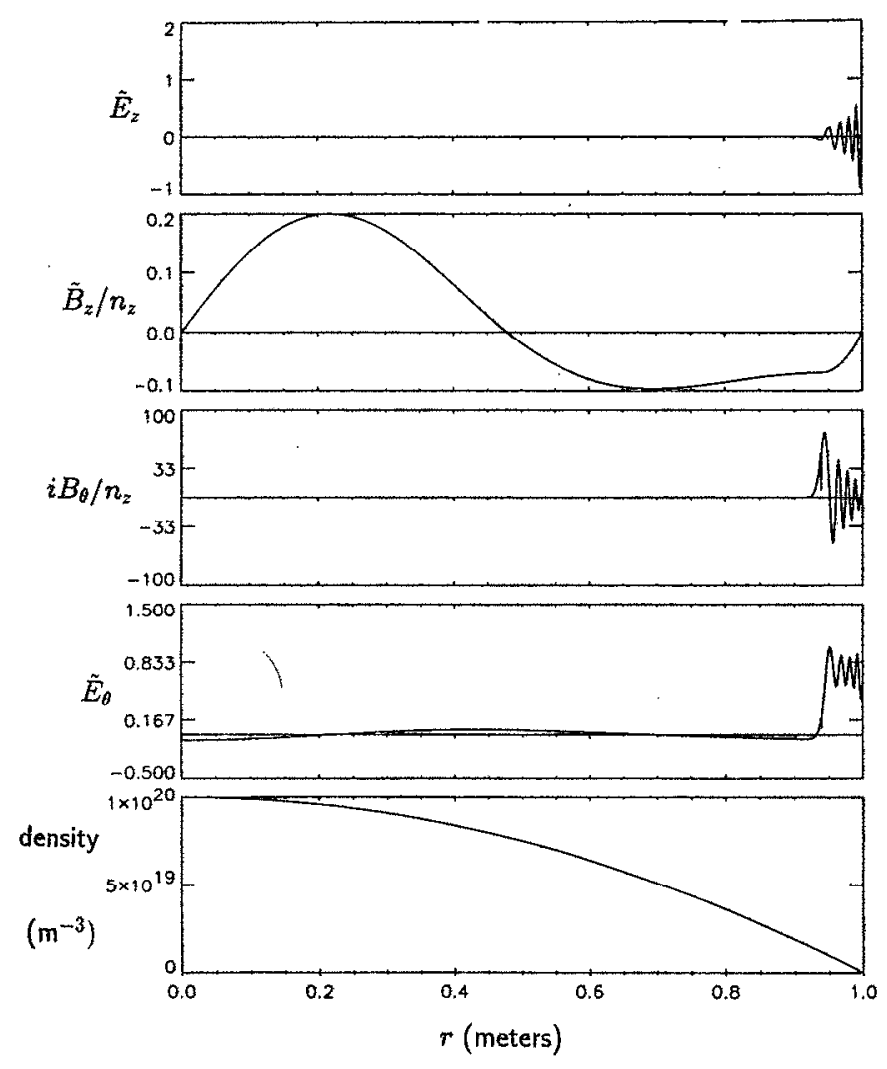

FIG. 3. Same as Fig. 2, but for parameters of a large tokamak; 50-50 D-T mixture, $f=10 \mathrm{MHz}, B=5 \mathrm{~T}, n_{z}=15, m=1, \vec{E}_{z}$ excitation.

$E$ wave approaches the Alfvén layer (going in the direction of increasing density), $\tilde{E}_{z}$ decreases in magnitude while $\tilde{B}_{y}$ increases. This is a counterexample to the common perception that all field components of a mode must increase or decrease in tandem. What is happening here is that the shear wave (which has both $\tilde{E}_{z}$ and $\tilde{B}_{y}$ components) is changing from being quasielectrostatic when it is on the low density side of the Alfvén layer to being inductive when it approaches the Alfvén layer.

Note that the perpendicular field components are finite at the Alfvén layer, but there are slight numerical 'glitches' in the Fig. 3 plots of these perpendicular components. These glitches come from trying to calculate the ratio zero/zero numerically and result from imperfect numerical cancellation of the two big terms analogous to the numerator terms of Eq. (149). The glitches could have been removed by using L'Hôpital's rule at the Alfvén layer, but it was decided to leave them in to show the accuracy of the solution and also to act as a marker for the Alfvén layer. In order to keep the glitches small, the radial derivatives of $\tilde{E}_{z}$ and $\tilde{B}_{z}$ were calculated to third-order numerical accuracy. The glitches are too small to appear in the Fig. 2 plots; this is because the longer wavelengths in Fig. 2 result in a greater relative numerical accuracy.

\section{RESONANCE CONE BEHAVIOR OF E WAVE}

\section{A. Derivation of resonance cone equation}

In the limit when $n_{z}^{2} \gg S$, Eq. (88) becomes

$$
n_{\perp}^{2} S+n_{z}^{2} P \approx 0
$$

which if 'un-Fourier' analyzed can be written as

$$
\frac{\partial^{2} \tilde{E}_{z}}{\partial x^{2}}+\frac{\partial^{2} \tilde{E}_{z}}{\partial y^{2}}=\left|\frac{P}{S}\right| \frac{\partial^{2} \tilde{E}_{z}}{\partial z^{2}} .
$$

This is a hyperbolic partial differential equation having solutions of the form

$$
\tilde{E}_{z}=\tilde{E}_{z}(z \pm \sqrt{|P / S|} r)
$$

where $r=\sqrt{x^{2}+y^{2}}$. This is called a resonance cone behavior and has been thoroughly studied ${ }^{44-50}$ in the lower and upper hybrid regimes, but has received little attention in the $\omega \ll \omega_{c i}$ regime. In the $\omega \ll \omega_{c i}$ regime the cone angle $\theta_{c}$ is given by

$$
\tan \theta_{c}=\frac{r}{z}=\sqrt{\left|\frac{S}{P}\right|} \approx \frac{c / v_{A}}{\omega_{p e} / \omega}=\frac{\omega}{\omega_{g m}}
$$

where $\omega_{g m}=\left|\omega_{c e} \omega_{c i}\right|^{1 / 2}$ is the geometric mean of the electron and ion cyclotron frequencies. Since $\omega_{c i} \leqslant \omega_{g m}$ and by assumption $\omega / \omega_{c i} \ll 1$, the cone angle is very small. For example if $\omega / \omega_{c i}=1 / 3$ and the plasma ions are hydrogen, then the cone angle will be $\theta_{c} \simeq 10^{-2}$ radian. Nevertheless, for large $z$ there will be significant propagation across the magnetic field, invalidating the statement that shear Alfyén waves only propagate along field lines and do not propagate across field lines. We note that Borg et al. ${ }^{51}$ have also discussed aspects of the resonance cone behavior of shear Alfvén waves.

Thus, a finite extent antenna located at the edge of an inhomogeneous plasma and aligned parallel to the $z$ axis will excite a disturbance having the shape of the image of the antenna field distribution and propagating into the plasma along the resonance cone trajectory. We call this the quasielectrostatic torsional Alfvén resonance cone (QETARC). If the antenna is infinite in the $y$ direction and located at $x_{s}$, this disturbance will have the simple form

$$
\begin{aligned}
\tilde{E}_{z}(x, z) \approx & \frac{1}{2}\left[\tilde{E}_{\text {ant }}\left(z-\theta_{c}^{-1}\left(x-x_{s}\right)\right)\right. \\
& \left.+\tilde{E}_{\text {ant }}\left(z+\theta_{c}^{-1}\left(x-x_{s}\right)\right)\right]
\end{aligned}
$$

where $\tilde{E}_{\text {ant }}(z)$ is the field distribution along the antenna. The cone trajectories are just characteristics of a hyperbolic partial differential equation in the $x-z$ plane. A plot of cone trajectories excited by a periodic source in the context of lower hybrid waves is given in Ref. 47; the Alfvén cone trajectories will be similar, except that the $k_{z}$ spectral components constituting the Alfvén resonance cone become electromagnetic in nature as $S \rightarrow n_{z}^{2}$ and reflect specularly from the $n_{z}^{2}=S$ layer (assuming $k_{y}=0$ so that there is no coupling between compressional and shear modes).

This resonance cone point of view is quite different from the conventional MHD point of view. In the conventional MHD view, the shear Alfvén dispersion is determined by Eq. (12) so that $k_{z}$ is determined by plasma parameters and waye 
frequency. In the resonance cone point of view (which we assert is the correct point of view), $k_{z}$ is not determined by the plasma, but is instead determined by the $z$-Fourier transform of the antenna; e.g. an antenna that is periodic in $z$ will excite a single $k_{z}$ whereas a localized antenna (delta function in $z$ ) will excite a broad $k_{z}$ spectrum. Each $k_{z}$ component excited by the antenna propagates according to Eq. (88), and what one observes is the sum of all these components. A localized source does not excite the single $k_{z}$ predicted by Eq. (12), but instead excites a broad spectrum of $k_{z}$. The part of the spectrum satisfying $n_{z}^{2} \gg S$ combine to form the resonance cone.

This resonance cone interpretation is consistent with experimental observations reported by Borg and Cross ${ }^{52}$ who used a localized antenna to excite shear Alfvén waves in a tokamak and measured the poloidal protile of the wave at a toroidal separation of $180^{\circ}$ from the transmitter. However, the angular resolution in their experiment was not small enough to be able to resolve the cone angle which would be of the order of a one degree angular displacement from $\mathbf{B}$.

The resonance cone nature and the electrostatic dispersion given by Eq. (154) have, however, been experimentally verified by $\mathrm{Ono}^{53}$ for the case of direct excitation at fixed $\omega$ by an electrostatic, finite extent antenna imposing $\tilde{E}_{z}$ with a well defined $k_{z}$ [although these electrostatic modes were clearly observed and identified with Eq. (154) in Ref. 53, the connection with shear Alfvén waves was not realized]. Skiff, Wong, and $\mathrm{Ono}^{54}$ have observed excitation of this electrostatic mode also, but via parametric decay from a higher frequency mode.

\section{B. Relationship of group velocity to resonance cones}

The $E$ wave dispersion can be written in unnormalized form as

$$
\omega^{2}=\frac{k_{z}^{2} v_{A}^{2}}{1+k_{x}^{2} c^{2} / \omega_{p e}^{2}}
$$

and the resonance cone limit corresponds to the case where $k_{z}^{2} v_{A}^{2} / \omega^{2} \gg 1$ so that the dispersion becomes simply $\omega=\omega_{g m} \cot \theta$. Let us now calculate the group velocity associated with Eq. (159) and see how this group velocity is related to the resonance cone.

Taking the derivative of Eq. (159) with respect to $\mathbf{k}$ gives

$$
\omega \frac{\partial \omega}{\partial \mathbf{k}}=\frac{k_{z} v_{A}^{2}}{1+k_{x}^{2} c^{2} / \omega_{p e}^{2}} \hat{z}-\frac{k_{z}^{2} v_{A}^{2} k_{x} c^{2} / \omega_{p e}^{2}}{\left[1+k_{x}^{2} c^{2} / \omega_{p e}^{2}\right]^{2}} \hat{x}
$$

so that we see that the direction of the $x$ component of the group velocity is in the $-k_{x}$ direction. Thus this wave is a backwards wave ${ }^{55}$ in the $x$ direction and, in order to have energy propagate into the plasma (positive $x$ direction), when we solve Eq. (159) for $k_{x}$ we must choose the negalive root for $k_{x}$. In this case Eq. (159) gives

$$
k_{x}=-\frac{\omega_{p e}}{c} \sqrt{\frac{k_{z}^{2} v_{A}^{2}}{\omega^{2}}-1} .
$$

Substituting for $k_{x}$ in Eq. (160) gives

$$
\frac{\partial \omega}{\partial \mathbf{k}}=\hat{z} \frac{\omega}{k_{z}}+\hat{x} c\left(\frac{\omega^{2}}{k_{z}^{2} v_{A}^{2}}\right) \frac{\omega}{\omega_{p e}} \sqrt{\frac{k_{z}^{2} v_{A}^{2}}{\omega^{2}}-1} .
$$

Thus, the parallel component of the group velocity is constant in an inhomogeneous slab plasma and is equal to the parallel phase velocity. The perpendicular component of the group velocity is small and goes to zero at the $\omega=k_{z} v_{A}$ layer resulting in specular reflection from the layer. Far from the layer, where $k_{z}^{2} v_{A}^{2} / \omega^{2} \gg 1$, the group velocity is approximately

$$
\frac{\partial \omega}{\partial \mathbf{k}} \approx \hat{z} \frac{\omega}{k_{z}}+\hat{x} c \frac{\omega^{2}}{\omega_{p e} k_{z} v_{A}} .
$$

In this case the tangent of the angle the group velocity makes with respect to the magnetic field is

$$
\frac{\hat{x} \cdot(\partial \omega / \partial \mathbf{k})}{\hat{z} \cdot(\partial \omega / \partial \mathbf{k})}=\frac{\omega}{\omega_{g m}}
$$

which is independent of $k_{z}$ so that all $k_{z}$ components have the same group velocity angle (this is what gives the resonance cone). Thus, far from the reflecting layer, the group velocity lies along the resonance cone. From Eqs. (154) and (163) we see that $\mathbf{k} \cdot \partial \omega / \partial \mathbf{k}=0$ so that the group and phase velocities are orthogonal (this is another way of seeing that the wave is backwards in the $x$ direction).

From Eq. (162) we see that at the $\omega=k_{z} v_{A}$ layer where reflection occurs, the group velocity is parallel to the magnetic field. Thus Alfvén's original analysis correctly describes the wave at the reflecting layer, but at no other value of $x$.

\section{Relation to hot electron low frequency resonance cones}

If the plasma parameters are such that $v_{T i}<\omega / k_{z}<v_{T e}$, then the electron parallel dielectric has a Boltzmann form, while the ions retain their cold plasma behavior. In this case the parallel dielectric becomes

$$
P=1+\frac{1}{k_{z}^{2} \lambda_{D e}^{2}}-\frac{\omega_{p i}^{2}}{\omega^{2}}
$$

If $\omega>k_{z} c_{s}$ (i.e., $n_{z}<c / c_{s}$ ) where $c_{s}=\omega_{p i} \lambda_{D e}$ is the ion acoustic velocity, then the middle term in Eq. (165) dominates so that $P$ is positive and one obtains a hot electron mode which propagates on the high density, low field side of the $\omega=k_{z} v_{A}$ layer.

However, for very short parallel wavelengths such that $n_{z}>c / c_{s}$ the last term in Eq. (165) dominates so that $P$ becomes negative again, suggesting the possibility of another resonance cone since once again $P$ and $S$ have opposite signs. Keeping finite $\omega / \omega_{c i}$ terms, the perpendicular dielectric is

$$
S \simeq 1-\frac{\omega_{p i}^{2}}{\omega^{2}-\omega_{c i}^{2}}
$$

since $\omega_{p i}^{2} / \omega_{c i}^{2} \gg \omega_{p e}^{2} / \omega_{c e}^{2}$. Using Eqs. (165) and (166), the electrostatic dispersion relation becomes 


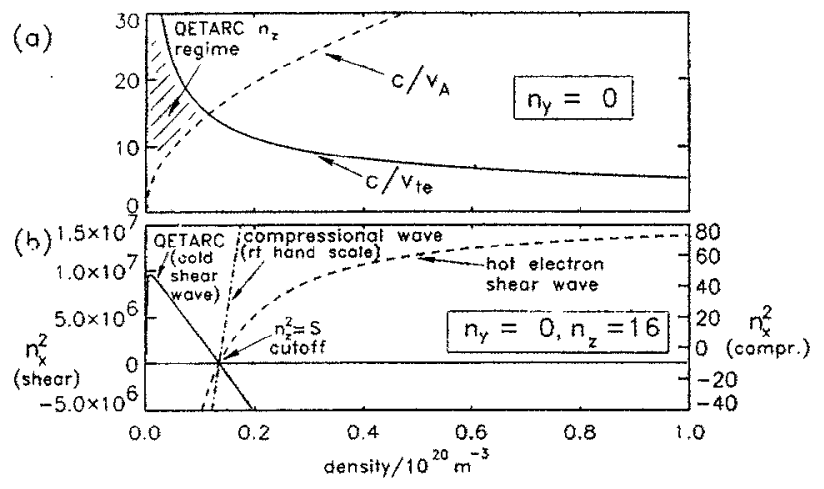

FIG. 4. (a) Plot of Eq. (175) for fusion reactor parameters described in text, shaded area shows allowed region of $E$ wave (also called quasielectrostatic torsional Alfvén resonance cone); (b) dispersion relations in limit $\omega / \omega_{c i} \rightarrow 0$ and $n_{v}=0$ showing $E$ wave [shear mode, Eq. (88)] propagating on low density side of $\bar{S}=0$ cutoff, $B$ wave [compressional mode, Eq. (89)] and hot electron mode on high density side.

$$
\frac{k_{x}^{2} S+k_{z}^{2} P}{k^{2}}=1+\frac{1}{k^{2} \lambda_{D e}^{2}}-\frac{\omega_{p i}^{2}}{\omega^{2}-\omega_{c i}^{2}} \frac{k_{x}^{2}}{k^{2}}-\frac{\omega_{p i}^{2}}{\omega^{2}} \frac{k_{z}^{2}}{k^{2}}=0 .
$$

Kuehl, ${ }^{56}$ Burrell, ${ }^{57}$ and Bellan ${ }^{58}$ have shown that Eq. (167) does indeed provide a new resonance cone regime, this time with cone angle $\theta_{c} \approx \sin ^{-1}\left(\omega / \omega_{c i}\right) ;$ Bellan ${ }^{58}$ has experimentally demonstrated the existence of these cones.

\section{Simple electrostatic derivation of cold plasma resonance cone}

By using the quasielectrostatic assumption (which is equivalent to the assumption that $n_{z}^{2} \gg S$ ), the low frequency resonance cone behavior can be derived directly: The electrostatic assumption involves assuming $\tilde{\mathbf{E}}=-\nabla \tilde{\phi}$ where $\tilde{\phi}$ is an electrostatic potential. Since $\omega \ll \omega_{c i}$, the fluid velocity $\tilde{\mathbf{u}}_{\sigma}$ for each plasma species $\sigma$ is the sum of the $\mathbf{E} \times \mathbf{B}$ velocity, the polarization drifts and the parallel quiver velocity,

$$
\tilde{\mathbf{u}}_{\sigma}=\frac{-\boldsymbol{\nabla} \tilde{\phi} \times \mathbf{B}}{B^{2}}+\frac{i \omega m_{\sigma}}{q_{\sigma} B^{2}} \nabla_{\perp} \tilde{\phi}+\frac{q_{\sigma}}{i \omega m_{\sigma}} \nabla_{\|} \tilde{\phi}
$$

The continuity equation for each species can be solved to give

$$
\tilde{n}_{\sigma}=\frac{\nabla \cdot\left(n_{\sigma} \tilde{\mathbf{u}}_{\sigma}\right)}{i \omega}
$$

while Poisson's equation is

$$
\nabla^{2} \tilde{\phi}=-\frac{1}{\epsilon_{0}} \sum_{\sigma=i, e} \tilde{n}_{\sigma} q_{\sigma} .
$$

We assume that $\nabla n \cdot \nabla \tilde{\phi} \times \mathbf{B}=\mathbf{0}$ which is equivalent to the $n_{y}=0$ assumption for our more general case. Using Eqs. (168), (169) in Eq. (170) gives the resonance cone equation

$$
\left.\nabla_{\perp} \cdot\left[\left(1+\frac{c^{2}}{v_{A}^{2}}\right) \nabla_{\perp} \tilde{\phi}\right]+\nabla_{\|} \cdot\left(1-\frac{\omega_{p e}^{2}}{\omega^{2}}\right) \nabla_{\|} \tilde{\phi}\right]=0
$$

which is equivalent to Eq. (155).

We note that resonance cones are a $\mathrm{cw}$ wave phenomena (i.e., require a sharp $\omega$ spectrum) and that if the antenna is pulsed (giving a broad $\omega$ spectrum) then, as shown in Ref. 59, a complicated wake-like, time-dependent spatial interference pattern is created in the plasma.

\section{TOROIDAL CURRENT DRIVE IN TOKAMAK PLASMAS}

In order to satisfy the cold plasma assumption and also avoid Landau damping the $E$ wave must satisfy (in unnormalized quantities)

$$
\omega / k_{z} \gg v_{T e}
$$

where $v_{T e}$ is the electron thermal velocity. In normalized quantities, this becomes

$$
\frac{c}{v_{T e}} \gg n_{z}
$$

The cold $E$ wave propagates on the low density, high field side of the $\omega=k_{z} v_{A}$ layer, i.e., for

$$
n_{z} \gg \frac{c}{v_{A}} .
$$

Thus, the allowed region for the $E$ wave is where

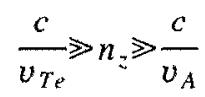

which will occur at the edge of the plasma where $v_{T e}$ is small and $v_{A}$ is large. Toroidal current drive ${ }^{60}$ will result if the $E$ wave is phased to have a unidirectional spectrum (like a lower hybrid grill ${ }^{61}$ antenna) and if $n_{z}$ is selected so that the $E$ wave Landau damps on electrons before it reaches the $\omega=k_{2} v_{A}$ layer. An indication of the allowed $n_{z}$ region in a tokamak reactor predicted by Eq. (175) is shown in Fig. 4(a). Here, for simplicity the electron temperature and density are assumed to have the same profile, so that it is possible to make a plot of $n_{z}$ versus density and have the temperature automatically parametrized. Both the $c / v_{r_{e}}$ and $c / v_{A}$ curves are shown in Fig. 4(a) and the allowed region predicted by Eq. (175) is shaded. Parameters relevant to a fusion reactor were chosen here, i.e., $B=5 \mathrm{~T}$ with a $50-50 \mathrm{D}-\mathrm{T}$ mix. The maximum electron temperature was assumed to be $10 \mathrm{keV}$, while the maximum density was assumed to be $10^{20} \mathrm{~m}^{-3}$.

Figure 4(a) indicates that for these parameters the maximum penetration for a Landau damped wave is obtained when $n_{z} \approx 16$. Choosing the wave frequency $f=\omega / 2 \pi=10$ $\mathrm{MHz}$ to satisfy the low frequency requirement $\omega \ll \omega_{c i}$ gives $\lambda_{\tilde{z}}=2 \pi / k_{\tilde{x}}=2 \mathrm{~m}$. For reference, Fig. 4(b) shows the dispersion relations of the $E$ wave (TM mode), the $B$ wave (TE mode) and the hot electron mode.

The polarization of the $E$ wave is such that the exciting antenna should generate an electric field parallel to the equilibrium magnetic field (i.e., equivalent to the finite $\tilde{E}_{z}$ of a slab system). The frequency regime is such that this excitation could be accomplished using an antenna inside the vacuum wall with $\tilde{J}_{2}$ currents to produce a traveling $\tilde{E}_{\|}$wave. This coupling is analogous to a toroidally segmented Ohmic heating transformer where each segment is driven by an RF generator phased so that the net field is a toroidal traveling wave. This current drive will be an edge drive but may still 
be useful since edge currents have been observed ${ }^{62}$ to penetrate anomalously towards the center of the plasma.

\section{SUMMARY AND CONCLUSIONS}

We have demonstrated that the logarithmic Alfvén 'resonance' singularity and associated localized absorption predicted by MHD do not exist in an ideal cold plasma. MHD fails because MHD ignores the $\tilde{E}_{z}$ field which is essential for describing the shear mode.

We have presented exact equations for cold plasma waves propagating across an inhomogeneous plasma and shown that in the low frequency (i.e., $\omega \ll \omega_{c i}$ ) regime, the $\omega=k_{z} v_{A}(x)$ layer acts as a cutoff for both TE and TM modes when $n_{y}=0$. However, a strong coupling between TE and TM modes occurs when $n_{y}$ is finite (but there is still no resonance). Numerical solutions of these equations for realistic situations illustrate the mode coupling and the nonexistence of logarithmic singularity and absorption. When $\omega / \omega_{c i}$ is finite and $n_{y}=0$ there is a region of inaccessibility surrounding the $\omega=k_{z} v_{A}(x)$ layer; this region disappears as $\omega / \omega_{c i} \rightarrow 0$.

In the low density, high field side of the $\omega=k_{z} v_{A}(x)$ layer the TM mode has a resonance cone, quasielectrostatic behavior (which cannot be described by ideal MHD); the cone angle is $\theta_{c}=\omega / \omega_{g m}$ where $\omega_{g m}$ is the geometric mean of the electron and ion cyclotron frequencies. This cone angle is typically very small so that waves excited by an external antenna will have a group velocity making an angle (the cone angle) that is very small with respect to the magnetic field, typically a small, but finite, fraction of a degree. These quasielectrostatic TM modes possess all the required attributes for toroidal current drive; the only limiting factor is drive will be an edge current drive. This should still be of interest if anomalous current penetration (observed on many experiments) takes place.

Note added in proof. The shear Alfvén resonance cone has also been observed experimentally by Gekelman et al. ${ }^{63}$

\section{ACKNOWLEDGMENT}

This work has been supported by United States Department of Energy Grant No. DE-FG-04-86ER53232.

${ }^{1}$ C. Uberoi, Phys. Fluids 15, 1673 (1972).

${ }^{2}$ J. Tataronis and W. Grossmann, Z. Phys. 261, 203 (1973); W. Grossmann and J. Tataronis, ibid. 261, 217 (1973).

${ }^{3} \mathrm{~A}$. Hasegawa and C. Uberoi, The Alfvén Wave (United States Department of Energy Technical Information Center, DOE/TIC -11191, 1982), available from National Technical Information Service, U.S. Department of Commerce, Springfield, Virginia, 22161.

${ }^{4}$ A. Hasegawa and L. Chen, Phys. Rev. Lett. 32, 454 (1974).

${ }^{5}$ L. Chen and A. Hasegawa, Phys. Fluids 17, 1399 (1974).

${ }^{6}$ J. P. Goedbloed, Phys. Fluids 18, 1258 (1975).

${ }^{7}$ T. H. Stix, Proceedings of the 2nd Varenna-Grenoble International Symposium on Heating in Toroidal Plasmas, Como, 1980 (Commision of the European Communities, Brussels, 1981), p. 631.

${ }^{8}$ T. H. Stix, Waves in Plasmas, 2nd ed. (American Institute of Physics, New York, 1992), pp. 32-33, 97, 354.

${ }^{9} \mathrm{~A}$. Hasegawa and L. Chen, Phys. Rev. Lett. 35, 370 (1975).

${ }^{10} \mathrm{~A}$. Hasegawa and L. Chen, Phys. Fluids 19, 1924 (1975).

"M. N. Rosenbluth and P. H. Rutherford, Phys. Rev. Lett. 34, 1428 (1975).

${ }^{12}$ E. Ott, J.-M. Wersinger, and P. Bonoli, Phys. Fluids 21, 2306 (1978).
${ }^{13}$ C. F. F. Karney, F. W. Perkins, and Y.-C. Sun, Phys. Rev. Lett. 42, 1621 (1979).

${ }^{14}$ Y. Li, S. Mahajan, and D. W. Ross, Phys. Fluids 30, 1466 (1987).

${ }^{15}$ R. Mett and J. B. Taylor, Phys. Fluids B 4, 73 (1992).

${ }^{16} \mathrm{~J}$. Vaclavik and K. Appert, Nucl. Fusion 31, 1945 (1991).

${ }^{17}$ R. Behn, A. de Chamrier, G. A. Collins, P.-A. Duperrex, A. Heym, F. Hofmann, Ch. Hollenstein, B. Joye, R. Keller, A. Lietti, J. B. Lister, J.-M. Moret, S. Nowak, J. O'Rourke, $\Lambda$. Pochelon, and W. Simm, Plasma Phys. Controlled Fusion 26, 173 (1984).

${ }^{18} \mathrm{M}$. J. Ballico and R. Cross, Plasma Phys. Controlled Fusion 33, 1841 (1991).

${ }^{19}$ R. Majeski, P. Probert, P. Moroz, T. Intrator, R. Breun, D. Brouchous, H. Y. E. Chen, J. R. DeKock, D. Diebold, M. Doczy, R. Fonck, N. Hershkowitz, R. D. Johnson, M. Kishinevksy, G. McKee, J. Meyer, P. Nonn, S. P. Oliva, J. Pew, J. Sorensen, T. Tanaka, M. Vukovic, and G. Winz, Phys. Fluids B 5, 2506 (1993).

${ }^{20}$ M. Cekic, B. A. Nelson, and F. Ribe, Phys. Fluids B 4, 392 (1992).

${ }^{21}$ A. Tsushima, Y. Amagashi, and M. Inutake, Phys. Lett. A 88, 457 (1982).

${ }^{22}$ I. J. Donnelly and B. E. Clancy, Aust. J. Phys. 36, 305 (1983).

${ }^{23}$ M. A. Lee and B. Roberts, Astrophys. J. 301, 430 (1986).

${ }^{24}$ J. V. Hollweg and G. Yang, Geophys. Res. 93, 5423 (1988).

${ }^{25}$ E. N. Parker, Astrophys. J. 376, 355 (1991).

${ }^{26}$ M. J. Thompson and A. N. Wright, J. Geophys. Res. 98, 15541 (1993).

${ }^{27}$ R. S. Steinolfson and J. M. Davila, Astrophys. J. 415, 354 (1993).

${ }^{28}$ F. D. Witherspoon, S. C. Prager, and J. C. Sprott, Phys. Rev. Lett. 53, 1559 (1984).

${ }^{29}$ See Ref. 8, Chap. 1.

${ }^{30}$ H. Alfvén, Ark. Mat. Astron. Fys. B 29, 2 (1943).

${ }^{31}$ E. Astrom, Ark. Fys. B 2, 42 (1950).

${ }^{32} \mathrm{~J}$. W. Dungy, Nature 1260, 1029 (1951).

${ }^{33}$ See Ref. 8 , p. 32.

${ }^{34}$ See Ref. 3, p. 95.

${ }^{35}$ V. E. Golant, Zh. Tekh. Fiz. 41, 2492 (1971) [Sov. Phys. Tech. Phys.-JETP 16, 1980 (1972)].

${ }^{36}$ See Ref. 8 , pp. $98-101$.

${ }^{37}$ See Ref. 8, pp. $26-29$.

${ }^{38}$ W. P. Allis, S. J. Buchsbaum, and A. Bers, Waves in Anisotropic Plasmas (MIT Press, Cambridge, MA, 1963), pp. 135, 170, 172.

${ }^{39}$ D. G. Swanson, Plasma Waves (Academic, San Diego, 1989), pp. 205212.

${ }^{40}$ W.-Q. Li, D. W. Ross, and S. M. Mahajan, Phys. Fluids B 1, 2353 (1989).

${ }^{41}$ See Ref. 39 , p. 53.

${ }^{42}$ G. M. Murphy, Ordinary Differential Equations and Their Solutions (Van Nostrand, Princeton, 1960), Item 194, p. 329.

${ }^{43}$ H. R. Lewis and P. M. Bellan, J. Math. Phys. 31, 2592 (1990).

${ }^{44}$ R. K. Fisher and R. W. Gould, Phys. Rev. Lett. 22, 1093 (1969); R. K. Fisher and R. W. Gould, Phys. Fluids 14, 857 (1971).

${ }^{45}$ R. J. Briggs and R. R. Parker, Phys. Rev. Lett. 29, 852 (1972).

${ }^{46} \mathrm{H}$. H. Kuehl, Phys. Fluids 16, 1311 (1973).

${ }^{47}$ P. M. Bellan and M. Porkolab, Phys. Rev. Lett. 34, 124 (1975); Phys. Fluids 17, 1592 (1974).

${ }^{48}$ R. L. Stenzl and W. Gekelman, Phys. Rev. A 11, 2057 (1975).

${ }^{49}$ P. M. Bellan and M. Porkolab, Phys. Fluids 19, 995 (1976).

${ }^{50}$ G. A. Wurden, K. L. Wong, and M. Ono, Phys. Fluids 28, 716 (1985).

${ }^{5 i}$ G. G. Borg, M. H. Brennan, R. C. Cross, L. Giannone, and I. J. Donnelly, Plasma Phys. Controlled Fusion 27, 1125 (1985).

${ }^{52}$ G. G. Borg and R. C. Cross, Plasma Phys. Controlled Fusion 29, 681 (1987).

${ }^{53}$ Pure He curve in Fig. 2(c) of M. Ono, Phys. Rev. Lett. 42, 1267 (1979).

${ }^{54}$ LF2 mode in F. N. Skiff, K. L. Wong, and M. Ono, Phys. Fluids 27, 2205 (1984).

${ }^{55}$ See Fig. 3 of Ref. 48 and Figs. 11 and 12 of Ref. 49 for examples of backwards wave propagation.

${ }^{56}$ H. H. Kuehl, Phys. Fluids 17, 1636 (1974).

${ }^{57}$ K. H. Burrell, Phys. Fluids 18, 897 (1975).

${ }^{58}$ P. Bellan, Phys. Rev. Lett. 37, 903 (1976).

${ }^{59}$ P. M. Bellan, Phys. Fluids 20, 649 (1977).

${ }^{60}$ N. J. Fisch, Phys. Rev. Lett. 41, 873 (1978).

${ }^{61}$ M. Brambilla, Nucl. Fusion 16, 47 (1976).

${ }^{62}$ M. Ono, G. J. Greene, D. Darrow, C. Forest, H. Park, and T. H. Stix, Phys. Rev. Lett. 59, 2165 (1987).

${ }^{63}$ W. Gekelman, D. Leneman, J. Maggs, and S. Vicena, "Experimental observation of Alfvén wave cones," to appear in Phys. Plasmas. 